\title{
EXTENSIONS AND DEGENERATIONS OF SPECTRAL TRIPLES
}

\author{
ERIK CHRISTENSEN, CRISTINA IVAN
}

\begin{abstract}
For a unital $\mathrm{C}^{*}$-algebra $\mathcal{A}$, which is equipped with a spectral triple $(A, H, D)$ and an extension $\mathcal{T}$ of $\mathcal{A}$ by the compacts, we construct a two parameter family of spectral triples $\left(A_{t}, K, D_{\alpha, \beta}\right)$ associated to $\mathcal{T}$.

Using Rieffel's notation of quantum Gromov-Hausdorff distance between compact quantum metric spaces it is possible to define a metric on this family of spectral triples, and we show that the distance between a pair of spectral triples varies continuously with respect the parameters. It turns out that a spectral triple associated to the unitarization of the algebra of compact operators is obtained under the limit - in this metric - for $(\alpha, 1) \rightarrow(0,1)$, while the basic spectral triple $(\mathcal{A}, H, D)$ is obtained from this family under a sort of a dual limiting process for $(1, \beta) \rightarrow(1,0)$.

We show that our constructions will provide families of spectral triples for the unitarized compacts and for the Podles̀ sphere. In the case of the compacts we investigate to which extent our proposed spectral triple satisfies Connes' 7 axioms for noncommutative geometry, [8].
\end{abstract}

\section{INTRODUCTION}

The so called Toeplitz algebra, say $\mathcal{T}$, may be obtained in a number of different ways. The most simple description of it is possibly as the $\mathrm{C}^{*}$-algebra on the Hilbert space $\ell^{2}(\mathbb{N})$ generated by the unilateral shift. A more profound description which relates to analysis, can be obtained via the algebra, $\mathcal{C}:=\mathrm{C}(\mathbb{T})$, of continuous functions on the unit circle. A function $f$ in this algebra is represented as a multiplication operator, $M_{f}$ on the Hilbert space $H:=L^{2}(\mathbb{T})$ of square integrable functions. This space has a subspace $H_{+}$, which consists of those functions in $H$ that have an analytic extension to the interior of the unit disk. Let $P_{+}$

Date: November 21, 2018.

1991 Mathematics Subject Classification. Primary, 58B34, 46L65 ; Secondary, 46L87, 83C65 .

Key words and phrases. spectral triple, non commutative compact metric space, degeneration of metrics, extension, $\mathrm{C}^{*}$-algebra, quantized calculus, Toeplitz algebra, Podles̀ sphere. 
denote the orthogonal projection of $H$ onto $H_{+}$, then the compression to $H_{+}$of a multiplication operator $M_{f}$ for a continuous function $f$ on $\mathbb{T}$ becomes the Toeplitz operator $T_{f}:=P_{+} M_{f} \mid H_{+}$, and these operators form a subspace in the Toeplitz algebra such that the Toeplitz algebra becomes the direct sum of $\left\{T_{f} \mid f \in \mathcal{C}\right\}$ and the algebra of compact operators on $H_{+}$. In this way the Toeplitz algebra becomes an extension of $\mathcal{C}$ by the compact operators. The mapping $\mathcal{C} \ni f \rightarrow T_{f}$ relates to the differentiable structure on the circle in the way that for the ordinary differentiation on the circle with respect to arc length, i. e. $D:=\frac{1}{i} \frac{d}{d \theta}$, we know that the space $H_{+}$is the closed linear span of the eigenvectors corresponding to non negative eigenvalues for $D$, so there is a strong connection between the differentiable structure on the circle and the operator theoretical construction called extension, of $\mathcal{C}$ by the compacts. In this article we will study this process from a more general point of view. Our study is based on Connes' notion of a spectral triple which is a way of expressing a differentiable structure in the world of non-commutative *-algebras, [7].

Definition 0.1. Let $\mathcal{A}$ be a unital $C^{*}$-algebra, $H$ a Hilbert space which carries a faithful unital representation $\pi$ of $\mathcal{A}$ and $D$ an unbounded self-adjoint operator on $H$. For a dense self-adjoint subalgebra $A$ of $\mathcal{A}$ the set $(A, H, D)$ is called a spectral triple associated to $\mathcal{A}$ if

(i) For all a in $A$ the commutator $[D, \pi(a)]$ is bounded and densely defined.

(ii) the operator $\left(I+D^{2}\right)^{-1}$ is compact.

In this article our starting point is a spectral triple associated to a $\mathrm{C}^{*}$-algebra $\mathcal{A}$ and we want to study some of the possibilities for constructing spectral triples associated to an extension of $\mathcal{A}$ by the algebra of compact operators on an infinite dimensional Hilbert space. Our fundamental example of a spectral triple is the one coming from the unit circle, as described above. This particular example was investigated by Connes and Moscovici in [9], where they constructed a spectral triple associated to the the Toeplitz algebra for each natural number $n$ in the following way. Let $S$ denote the unilateral shift on $H_{+}$, i. e. for the canonical basis for $H_{+}$we have $S e_{k}=e_{k+1}$, and let $D_{+}=-i \frac{d}{d \theta} \mid H_{+}$, which means that $D_{+}$is the positive self-adjoint operator on $H_{+}$which satisfies $D_{+} e_{k}=k e_{k}$. Then the Hilbert space $K$ of the spectral triple for the Toeplitz algebra is defined as $K=H_{+} \oplus H_{+}$, and the Dirac operator $D_{n}$ is defined via the matrix form

$$
D_{n}:=\left(\begin{array}{cc}
0 & D_{+} S^{n} \\
\left(D_{+} S^{n}\right)^{*} & 0
\end{array}\right) .
$$


In the construction we present in this paper we look at a spectral triple $(A, H, D)$ associated to a $\mathrm{C}^{*}$-algebra $\mathcal{A}$ and an orthogonal projection $P$ onto a subspace of $H$ such that $P$ commutes with $D$, and for each operator $a$ from $\mathcal{A}$ the commutator $[P, a]$ is compact. All of this set-up is analogous to the classical spectral triple for the circle algebra, but there is, in general, no counterpart to the unilateral shift. This means that we have to modify the construction by Connes and Moscovici in order to construct a spectral triple associated to the $\mathrm{C}^{*}$-algebra generated by the operators $\{P a|P H| a \in \mathcal{A}\}$ and the compact operators on $P H$. A $\mathrm{C}^{*}$-algebra, obtained this way, is called an extension of $\mathcal{A}$ by the compacts, and one of the problems we try to solve in this article is to find ways to extend a spectral triple associated to a $\mathrm{C}^{*}$-algebra to a spectral triple for an extension of that algebra by the compacts. A more general question of this sort has been studied by Chakraborty in [6]. In that paper he studies compact quantum metric spaces as introduced by Rieffel [28], and he investigates the possibilities to generate the structure of a compact quantum metric space associated to an extension of a $\mathrm{C}^{*}$-algebra which is associated to the given compact quantum metric space. In Chakraborty's article he studies a short exact sequence of $\mathrm{C}^{*}$-algebras of the type

$$
0 \rightarrow \mathcal{K} \otimes \mathcal{A} \rightarrow \mathcal{A}_{1} \rightarrow \mathcal{A}_{2} \rightarrow 0
$$

for which the last homomorphism has a positive splitting $\sigma: \mathcal{A}_{2} \rightarrow$ $\mathcal{A}_{1}$, and he shows that if there is a compact quantum metric space associated to both $\mathcal{A}$ and $\mathcal{A}_{2}$, then there exist several compact quantum metric spaces associated to the $\mathrm{C}^{*}$-algebra $\mathcal{A}_{1}$. This set up is more general than ours from the point of view of possible extensions, but our concern is spectral triples rather than the construction of compact quantum metric spaces. Chakraborty offers several applications of his construction to known examples, such as the Podles̀ sphere. We show that our construction can also be applied to generate spectral triples for this example and also for the algebra of compact operators on a separable Hilbert space.

We will, through the entire article, suppose that $(A, H, D)$ is a spectral triple associated to a unital $\mathrm{C}^{*}$-algebra $\mathcal{A}$, which is a subalgebra of $B(H)$. As in the book [16] Definition 2.7.7 and Chapter 5, we will study extensions of Toeplitz type. This means that we are interested in an orthogonal projection $P$ in $B(H)$ which commutes with $\mathcal{A}$ modulo the compact operators. One can then define a $\mathrm{C}^{*}$-algebra $\mathcal{B}$ on $P H$ as the $\mathrm{C}^{*}$-algebra generated by the space of operators $\{P a|P H| a \in \mathcal{A}\}$ in $B(P H)$. For each operator $a$ in $\mathcal{A}$ we have that $(I-P) a \mid P H$ is compact, so operators of the form $P a^{*}(I-P) a \mid P H$ are compact and 
- unless $P$ commutes with $\mathcal{A}$ - the algebra $\mathcal{B}$ will contain non trivial compact operators. We will let $C(P H)$ denote the algebra of compact operators on $P H$. In the classical case of the Toeplitz algebra for the circle we actually have $C(P H)=\mathcal{B} \cap C(P H)$. In any case, independently of what the $\mathrm{C}^{*}$-algebra $\mathcal{B} \cap C(P H)$ might be we will consider the $\mathrm{C}^{*}$-algebra $\mathcal{T}$ which is defined as the sum $\mathcal{T}:=\mathcal{B}+C(P H)$.

Let $\mathcal{Q}(P H)$ denote the Calkin algebra $B(P H) /(C P H)$ and let $\kappa$ denote the quotient homomorphism, then we can define a homomorphism $\varphi$ of $\mathcal{A}$ into $\mathcal{Q}(P H)$ by $\varphi(a):=\kappa(P a \mid P H)$. The extensions we will consider are those obtained via the construction described above which also have the property that the homomorphism $\varphi$ is faithful on $\mathcal{A}$. We may then define a homomorphism of $\mathcal{T}$ onto $\mathcal{A}$ as $\varphi^{-1} \circ \kappa$, and we will say that a projection $P$ in $B(H)$ which satisfies all the properties discussed here is of Toeplitz type. We will not study all such projections, but restrict our investigations to projections of Toeplitz type such that $P$ commutes with the Dirac operator $D$ and satisfies the following regularity property

$$
\forall a \in A \quad[P D, a] \quad \text { is bounded and densely defined. }
$$

Under these assumptions we will say that the quadruple $((A, H, D), P)$ is of Toeplitz type.

We would like to remind the reader that for any spectral triple of infinite dimensions - like $(A, H, D)$ the spectral projection $P_{+}$for $D$ corresponding to the interval $[0, \infty[$ is a natural candidate for $P$. This follows from the well known fact that the symmetry $2 P_{+}-I$ generates a bounded Fredholm module [3]. In the case $P=P_{+}$the regularity condition amounts to the assumption that for any $a$ from $A$, the commutator $[|D|, a]$ is bounded, too.

We can now describe the general construction, which we will study here. So for a quadruple $((A, H, D), P)$ of Toeplitz type we define the $\mathrm{C}^{*}$-algebra $\mathcal{T}$, as above, a Hilbert space $K:=P H \oplus H$ and a representation $\pi$ of $\mathcal{T}$ on $K$ given by the matrix form

$$
\pi(t):=\left(\begin{array}{cc}
t & 0 \\
0 & \varphi^{-1}(\kappa(t))
\end{array}\right)
$$

The Hilbert space $K=P H \oplus H$ decomposes as $K=P H \oplus P H \oplus$ $(I-P) H$ and we can see that the first two summands are exactly analogous to the ones appearing in the Connes-Moscovici construction. We have tried to follow their idea, but our analysis indicates that we can not in general give up the information which is encoded in the $(I-P) H$ part of the structures, so we will consider $K=P H \oplus H$ 
rather than $P H \oplus P H$. On the other hand this opens the possibility to play on the two parts with different weights as the introduction of parameters in our proposal for a Dirac operator shows. Since $D$ is supposed to commute with $P$, the regularity conditions imposed make it possible to define a family of Dirac operators on $K$ in the following way. For positive reals $\alpha, \beta$ such that $\alpha \beta \leq 1$ we define an unbounded self-adjoint operator $D_{\alpha, \beta}$ on $K$ via its matrix:

$$
D_{\alpha, \beta}:=\left(\begin{array}{ccc}
0 & \beta D \mid P H & 0 \\
\beta D \mid P H & \frac{1}{\alpha} D \mid P H & 0 \\
0 & 0 & \frac{1}{\alpha} D \mid(I-P) H
\end{array}\right)
$$

The reason for having the parameter $\alpha$ appearing in the form $1 / \alpha$ is mainly aesthetical. For instance, the formula giving a distance estimate between the non-commutative spaces obtained for two pairs of parameters, say $(\alpha, \beta)$ and $(\gamma, \delta)$ becomes by Theorem 3.9

$$
\left(\max \left\{\frac{\alpha \beta}{\gamma \delta}, \frac{\gamma \delta}{\alpha \beta}\right\}-1+\left|1-\frac{\beta}{\delta}\right|\right) \operatorname{diam}_{\alpha, \beta},
$$

and in this formula the product $\alpha \beta$ fits in as a parameter.

The reason why the parameters are supposed to satisfy the inequality $\alpha \beta \leq 1$ comes originally from the classical Toeplitz case, where it is quite easy to analyze the situation in details. It turns out that for this example and a pair of parameters $(\alpha, \beta)$ for which $\alpha \beta>1$ all the aspects of the non-commutative space associated to these parameters is already contained in the space given by the parameters $(1 / \beta, \beta)$. The general case does not work exactly in the same way, but the Remark 1.10, explains why we think nothing essential is lost, if we just stick to the region in the parameter space where $\alpha \beta \leq 1$. Still another argument for the choice of parameter space is that it turns out that the limiting process $\alpha \rightarrow 0$ behaves uniformly nice on the set of parameters where $\beta \geq \beta_{0}$. For the convergence $(\alpha, 1) \rightarrow(0,1)$ we find that it induces a convergence with respect to the quantum Gromov-Hausdorff metric of the compact quantum metric spaces associated to $\mathcal{T}$ for the parameters $(\alpha, 1)$ onto that of the unitarized algebra $\tilde{\mathcal{C}}$. Our intuitive description of this phenomenon is as follows. We think that the spectral triple acts like a microscope where we have a fixed screen to watch, but we are allowed to change the magnification. The parameter $\alpha$ is a measure of the actual size, say in meters, of the objects we can watch on our screen, and then $1 / \alpha$ is the magnification factor. When $\alpha$ decreases to zero, we loose the sight of the big picture and can only see tiny details of very small things. In the end - when $\alpha=0$ - our mathematical construction can only see the compacts, and they are considered to be 
the infinitesimals in Connes' dictionary [8]. The precise mathematical content of this story is contained in Theorem 4.6.

The limit $(1, \beta) \rightarrow(1,0)$ is quite easy to understand if you take a look at the definition of $D_{\alpha, \beta}$ just above, and you can see that you get the basic spectral triple $(A, H, D)$ back, but now in a degenerated representation. This is not a limit with respect to the quantum GromovHausdorff metric on the associated non commutative spaces, but rather a sort of degenerated limit where the compacts i. e. the infinitesimals become invisible. We can provide a simple 2-dimensional model of the picture we try to present. Look at the unit square $[0,1]^{2}$ in $\mathbb{R}^{2}$ and equip it with the metric $d_{\alpha, \beta}((x, y),(s, t)):=\alpha|x-s|+1 / \beta|y-t|$, then the limit $(\alpha, 1) \rightarrow(0,1)$ gives the unit interval $\{(0, y) \mid 0 \leq y \leq 1\}$ with its standard metric as the limit in the Gromov-Hausdorff metric. For $(1, \beta) \rightarrow(1,0)$ there is no limit of this sort but we get - pointwise - a degenerate metric $d_{1,0}$ on the unit square as a limit. This degenerate metric is given by the formula

$$
d_{1,0}((x, y),(s, t))=\left\{\begin{array}{ll}
|x-s| & \text { if } t=y \\
\infty & \text { if } t \neq y
\end{array} .\right.
$$

In the first version of the article we considered this limiting process as a sort of deformation, but we have been told by several people that we do not deform a product, so the wording is wrong. By looking into the literature on metric spaces associated to Riemannian structures we have found that the limiting processes we are watching may be considered as degenerations of metric spaces, so the title has been changed accordingly. Usually this sort of degeneration of Riemannian structures is studied under some assumptions on boundedness of curvature during the process $[4,5,15]$, but this last aspect of degeneration of metric structures does not apply to our results, at least for the time being.

On the other hand we still think that the limiting process $(1, \beta) \rightarrow$ $(1,0)$ - in the case where the algebra $\mathcal{A}$ is commutative - offers a way of describing a passage from a non-commutative compact metric space into a commutative compact metric space. The equation (2) indicates that a better description of the degeneration occurring while $\beta \rightarrow 0$ might possibly be; a passage from a non-commutative space to an infinite collection of disjoint identical copies of the same commutative space.

After we had posted the first pre-print version of this article on the arXiv, we were informed by Hanfeng Li, that our constructions can work in the settings of David Kerr's, [17], and his own, [22], and the one from their joint work, [18], where the quantum Gromov-Hausdorff 
metric is extended in a way which is based on the operator space structure of the given algebra. The introduction of the state spaces of the tensor products of the given algebra by the algebras of $n \times n$ complex matrices into the definition of a compact quantum space, is to be able to describe certain aspects of order in more details. We have chosen not to expand the present paper and hope that these results of Li's some day will find a suitable place to be presented.

Near the end we give a couple of examples and show that our method creates an abundance of spectral triples for the unitarized compact operators on a separable infinite dimensional Hilbert space. Then we show that the method, when applied to the unit circle and the classical differential operator $\frac{1}{i} \frac{d}{d \theta}$, gives a spectral triple associated to the classical Toeplitz $\mathrm{C}^{*}$-algebra. Based on this spectral triple we can then by a slight modification of our method obtain a spectral triple for the Podles̀ sphere. Unfortunately we can see no relations between our constructions and the ones presented in [10] and [11].

At the very end we present some small comments on the relations between the constructions in this article to the concepts of even and odd spectral triples and to analytic K-homology as described by Higson and Roe in their book [16]. It may be that further assumptions or conditions on the starting spectral triple may be used to give a basis for a more detailed study of such relations. In this paper we have been focusing on the quite general degeneration aspects of the extended spectral triples.

We are most thankful to the referee who has pointed out some problems in the first version of the article, and he has also suggested several possibilities for improvements. Among the questions he asked is the question on how the spectral triples constructed here relate to the 7 axioms for non-commutative geometry which Connes lists in the article [8]. To answer this question we have studied this question for our examples involoving the Toeplitz algebra and the unitarized compacts. The Toeplitz case seems not promising at all with respect to this investigation, so we have not included any comments on this aspect for the Toeplitz algebra. For the compacts we do check all the axioms, and we show that we can meet some, whereas for others we can not decide, but for the so-called reality axiom we get one of the signs wrong.

\section{A FAMILY OF SPECTRAL TRIPLES ASSOCIATED TO AN EXTENSION}

We will keep a $\mathrm{C}^{*}$-algebra $\mathcal{A}$ with an associated spectral triple $(A, H, D)$ fixed during the whole article and moreover suppose that $\mathcal{A}$ is a concrete $\mathrm{C}^{*}$-algebra acting on the Hilbert space $H$. As stated in the introduction, we will assume that we have a projection $P$ in $B(H)$ 
of Toeplitz type and study an extension $\mathcal{T}$ of $\mathcal{A}$ by the algebra $C(P H)$ of compact operators on $P H$. It should be remarked that we do not assume that the algebra of compact operators $C(P H)$ is contained in the $C^{*}$-algebra $\mathcal{B}$ generated by operators of the form $P a \mid P H$, and in particular we can also study the situation where $P$ commutes with $\mathcal{A}$. We will collect the definitions from the introduction in a formal definition.

Definition 1.1. Let $\mathcal{A}$ be a unital $C^{*}$-algebra on a Hilbert space $H$ and let

$(A, H, D)$ be a spectral triple associated to $\mathcal{A}$. A projection $P$ in $B(H)$ is said to be of Toeplitz type for $(A, H, D)$ if

(i) The projection $P$ commutes with $D$.

(ii) The projection $P$ commutes modulo the compacts with $\mathcal{A}$.

(iii) The homomorphism $\varphi$ of $\mathcal{A}$ to the Calkin algebra $\mathcal{Q}(P H)$, defined by $\varphi(a):=P a \mid P H+C(P H)$, is faithful.

For such a triple and a projection $P$ of Toeplitz type we define the Toeplitz extension $\mathcal{T}$ of $\mathcal{A}$ by $C(P H)$ as the $C^{*}$-algebra generated by

$$
\{P A|P H| A \in \mathcal{A}\} \cup C(P H)
$$

At the end of the paper we construct an example which will give a spectral triple for the Podles' sphere. That example is based on a slight variation of the construction presented in this paper, and it suggests that it might be possible to study extensions of $\mathcal{A}$ by a sub $\mathrm{C}^{*}$-algebra of $C(P H)$ instead. A generalization of our construction to cover cases like this seems possible, but also quite demanding with respect to extra details, so we have chosen only to consider extensions by all of $C(P H)$, and then just present the other point of view in connection with the example for the Podles' sphere.

Given a projection $P$ of Toeplitz type for $(A, H, D)$, we assume that $P$ commutes with $D$ and by this we mean that $P$ commutes with all the spectral projections of $D$. From this it follows that the unitary $S:=P-(I-P)$ also will commute with $D$ and $S$ will map the domain of definition for $D$ onto itself, ( [24], Proposition 5.3.18.) Hence the domain of definition for $D$ splits into a direct sum of its intersections with $P H$ and $(I-P) H$ respectively. We will need that the commutators from the spectral triple respect this decomposition too, and this is the basis for the following definition. In the classical case where $P=P_{+}$this means that we will not only demand that commutators $[D, a]$ are bounded and densely defined for $a$ in $A$, but we want both $[D, a]$ and $[|D|, a]$ to be bounded and defined on a common dense domain. 
Definition 1.2. A quadruple $((A, H, D), P)$ where $P$ is a projection of Toeplitz type for $(A, H, D)$, is said to be of Toeplitz type if:

(i) For any $a$ in $A$, the commutators $[P D, a]$ and $[(I-P) D, a]$ are bounded and densely defined and their common domain of definition contains two subspaces

$$
\operatorname{dom}([D, a]) \cap P H \text { and } \operatorname{dom}([D, a]) \cap(I-P) H
$$

which are dense in $P H$ and $(I-P) H$ respectively.

(ii) The operator $D_{P}:=D \mid P H$ has trivial kernel.

The properties in the definition above seem natural in the setting for a classical Toeplitz algebra, except for the last one. On the other hand that one does not really matter. Let namely $N$ denote the orthogonal projection onto the kernel of $D$, then $N$ is of finite rank, and since it is a spectral projection for $D$, it commutes with $P$ and we can replace $P$ by $P-P N$, without disturbing any properties of the extension we are studying. The first condition has been imposed in order to be able to look at commutators of the form $[P D, a] \mid P H$ and their relatives with restrictions to $(I-P) H$ and/or $P D$ replaced by $(I-P) D$. The conditions are made such that the lemma below holds. To keep the notational problems at a minimum we introduce the conventions that

$$
\begin{aligned}
H_{p} & :=P H, \quad H_{q}:=(I-P) H, \\
P_{p} & :=P, \quad P_{q}:=(I-P), \\
D_{p} & :=D P, \quad D_{q}:=D(I-P) .
\end{aligned}
$$

Lemma 1.3. For any $a$ in $A$ and any combination of the symbols $s, t, r$ in the set $\{p, q\}$

The closure of $\left(P_{s}[D, a] \mid H_{t}\right)=P_{s}$ the closure of $([D, a]) \mid H_{t}$

The closure of $\left(P_{s}\left[D_{r}, a\right] \mid H_{t}\right)=P_{s}$ the closure of $\left(\left[D_{r}, a\right]\right) \mid H_{t}$

Proof. We will not prove all these statements but restrict ourselves to the relation (3) in the situation where $s=p$ and $t=q$. The closure of the commutator $[D, a]$ is bounded and we will denote its closure by $\delta(a)$. It is immediate that as operators we have the inclusion $P[D, a] \mid(I-$ $P) H \subseteq P \delta(a) \mid(I-P) H$, and in order to show the statement of the lemma it is sufficient to show that $P[D, a] \mid(I-P) H$ is densely defined, but this is fulfilled by the condition (i) in Definition 1.2. We now claim that we can perform exactly the same computations with respect to any other combination of the symbols $\{p, q\}$, and then obtain the lemma. 
The effect of the lemma is that we may decompose the commutator $[D, a]$ into its matrix parts with respect to the decomposition of $H=$ $H_{p} \oplus H_{q}$, such that each of the 4 the matrix entries of the closure is the closure of the corresponding operator-theoretical matrix entry. From this follows the lemma just below:

Lemma 1.4. For any $a$ in $A$ the operators $D P_{p} a P_{q}$ and $D P_{q} a P_{p}$ are bounded and everywhere defined.

Proof. We remind you that a product of operators of the form $C B$ where $C$ is closed and $B$ is bounded is automatically closed, so if it is bounded it must be everywhere defined.

We will now define various maps and a spectral triple associated to $\mathcal{T}$. Before we give the definition we would like to mention that its first item is legal, due to a general result on ideals in $\mathrm{C}^{*}$-algebras that we recall here ([12], Corollary 1.5.6).

Proposition 1.5. Suppose that $\mathcal{I}$ is a two sided closed ideal of a $C^{*}$ algebra $\mathcal{A}$, and that $\mathcal{B}$ is a sub $C^{*}$-algebra of $\mathcal{A}$. Then $\mathcal{B}+\mathcal{I}$ is a $C^{*}$-algebra and

$$
\mathcal{B} /(\mathcal{B} \cap \mathcal{I}) \simeq(\mathcal{B}+\mathcal{I}) / \mathcal{I}
$$

is $a^{*}$-isomorphisms.

Let now $\kappa$ denote the quotient mapping of $B(P H)$ onto the Calkin algebra $\mathcal{Q}(P H)$, then the proposition above has the following corollary as a consequence.

Corollary 1.6. For any quadruple $((A, H, D), P)$ of Toeplitz type with associated Toeplitz extension $\mathcal{T}$, the images $\kappa(\mathcal{T})$ and $\varphi(\mathcal{A})$ in the Calkin algebra $\mathcal{Q}(P H)$ agree and $\kappa(\mathcal{T})$ is isomorphic to $\mathcal{A}$.

Definition 1.7. Let $((A, H, D), P)$ be a quadruple of Toeplitz type associated to a $C^{*}$-algebra $\mathcal{A}$. For the induced Toeplitz extension $\mathcal{T}$ of $\mathcal{A}$ we define:

(i) A representation $\rho: \mathcal{T} \rightarrow B(H)$ by $\rho(t):=\varphi^{-1}(\kappa(t))$.

(ii) A completely positive unital and injective mapping $T: \mathcal{A} \rightarrow \mathcal{T}$ by $T(a):=P a \mid P H$.

(iii) A projection $\Theta$ of $\mathcal{T}$ onto $C(P H)$ by $\Theta(t):=t-T(\rho(t))$.

(iv) For any $x$ in $B(H)$ and any combination of the symbols $s, r \in$ $\{p, q\}$ we define $x_{s r}$ in $B\left(H_{r}, H_{s}\right)$ by $x_{s r}:=P_{s} x \mid H_{r}$.

It should be noted that for an $a$ from $\mathcal{A}$, we have $T(a)=a_{p p}$.

Given a situation as above, we will then define a representation $\pi$ of $\mathcal{T}$ on a Hilbert space $K$ and a family of unbounded self-adjoint 
operators $D_{\alpha, \beta}$ on $K$, but it is not immediate that we will get spectral triples this way, so we start by defining the ingredients separately and study some of their properties.

Definition 1.8. Let $((A, H, D), P)$ be a quadruple of Toeplitz type associated to the $C^{*}$-algebra $\mathcal{A}$ and let $\mathcal{T}$ denote the induced Toeplitz algebra on the space PH. To this quadruple is associated:

(i) A dense self-adjoint subalgebra $A_{c}$ of $C(P H)$ defined by

$$
A_{c}:=\left\{k \in C(P H) \mid D_{p} k \text { and } k D_{p} \text { are bounded }\right\}
$$

(ii) $A$ dense self-adjoint subalgebra $A_{t}$ of $\mathcal{T}$ defined by

$$
A_{t}:=\left\{T(a)+k \mid a \in A, k \in A_{c}\right\}
$$

(iii) A Hilbert space $K$ defined as the sum

$$
K:=P H \oplus H=H_{p} \oplus H_{p} \oplus H_{q}
$$

(iv) $A$ representation $\pi$ of $\mathcal{T}$ on $K$ defined by

$$
\forall t \in \mathcal{T}: \quad \pi(t):=\left(\begin{array}{cc}
t & 0 \\
0 & \rho(t)
\end{array}\right) .
$$

(v) For positive reals $\alpha, \beta$ a self-adjoint operator $D_{\alpha, \beta}$ is defined on $K$ via its matrix, which, with respect to the decomposition $K=H_{p} \oplus H_{p} \oplus H_{q}$, is given by

$$
D_{\alpha, \beta}:=\left(\begin{array}{ccc}
0 & \beta D_{p} & 0 \\
\beta D_{p} & \frac{1}{\alpha} D_{p} & 0 \\
0 & 0 & \frac{1}{\alpha} D_{q}
\end{array}\right) .
$$

It may not be obvious that the linear space $A_{t}$ is an algebra, but it follows from Lemma 1.4.

We will show that for each pair $(\alpha, \beta)$ we will get a spectral triple for the Toeplitz extension $\mathcal{T}$ of $\mathcal{A}$, induced by the projection $P$. This will be an odd spectral triple and it is possible - via a standard trick - to obtain an even triple instead. But from the point of view we are studying here, namely the variation of the compact quantum metric spaces with respect to the parameters $\alpha$ and $\beta$ we do not get any changes if the investigation is performed with the odd spectral triple described above or an even one.

The properties of a quadruple of Toeplitz type now come into play and it helps us to split a commutator $\left[D_{\alpha, \beta}, \pi(t)\right]$ for a $t$ in $A_{t}$ into its matrix parts.

Lemma 1.9. For $a$ in $A$ and $k$ in $A_{c}$ and positive reals $\alpha, \beta$ with $\alpha \beta \leq 1$ the commutator $\left[D_{\alpha, \beta}, \pi((T(a)+k)]\right.$ is bounded. For each matrix part of the closure of this commutator, with respect to the decomposition $K=$ 
$P H \oplus P H \oplus(I-P) H$, the element is the closure of the corresponding matrix part of the algebraic commutator.

Proof. We will do the computations where they are defined purely algebraically, then show that each matrix entry is bounded and densely defined and then conclude that the closure of the commutator is the closure of the operator composed of the matrix entries. The reason why this is possible is the regularity assumptions and the Lemma 1.3.

$$
\begin{aligned}
& {\left[D_{\alpha, \beta}, \pi(T(a)+k)\right]} \\
& =\left[\left(\begin{array}{ccc}
0 & \beta D_{p} & 0 \\
\beta D_{p} & \frac{1}{\alpha} D_{p} & 0 \\
0 & 0 & \frac{1}{\alpha} D_{q}
\end{array}\right),\left(\begin{array}{ccc}
T(a)+k & 0 & 0 \\
0 & T(a) & a_{p q} \\
0 & a_{q p} & a_{q q}
\end{array}\right)\right] \\
& =\beta\left(\begin{array}{ccc}
{\left[D_{p}, T(a)\right]+D_{p} k} & {\left[D_{p}, T(a)\right]-k D_{p}} & D_{p} a_{p q} \\
-a_{q p} D_{p} & 0 & 0 \\
0 & 0
\end{array}\right) \\
& +\frac{1}{\alpha}\left(\begin{array}{ccc}
0 & 0 & 0 \\
0 & {[D, a]} \\
0 & {[D,}
\end{array}\right) .
\end{aligned}
$$

The lemma follows.

Remark 1.10. The idea in the setup of the commutator

$$
\left[D_{\alpha, \beta}, \pi(T(a)+k)\right]
$$

is that it shall reflect both of the given commutators $\left[D_{p}, k\right]$ and $[D, a]$ in such a way that a variation of the parameters $\alpha$ and $\beta$ will reveal information on each of these parts separately. Since

$$
\begin{aligned}
{\left[D_{p}, T(a)\right] } & =P[D, \pi(a) \mid P H \text { we get } \\
\left\|\left[D_{p}, T(a)\right]\right\| & \leq\|[D, \pi(a)]\| \text { and then for } \alpha \beta \leq 1 \\
\beta\left\|\left[D_{p}, T(a)\right]\right\| & \leq \frac{1}{\alpha}\left\|\left[D_{p}, T(a)\right]\right\| \leq \frac{1}{\alpha}\|[D, \pi(a)]\|
\end{aligned}
$$

Hence for $\alpha \beta \leq 1$ the term $\beta\left[D_{p}, T(a)\right]$ will not be of significance and then we see from (5) that in this case we will have

$$
\begin{aligned}
\frac{1}{\alpha}\|[D, \pi(a)]\| & \leq 1 \text { and } \max \beta\left\{\left\|D_{p} k\right\|,\left\|k D_{p}\right\|\right\} \leq 2 \text { if } \\
\left\|\left[D_{\alpha, \beta}, \pi(T(a)+k)\right]\right\| & \leq 1 .
\end{aligned}
$$


We will then impose the condition $\alpha \beta \leq 1$ in all of our future statements, and this also fits nicely with the results of Theorem 3.9 which indicate that the product $\alpha \beta$ is a relevant parameter.

Proposition 1.11. For any pair of positive real numbers $\alpha, \beta$ such that $\alpha \beta \leq 1$ the tuple

$$
\left(A_{t}, K, D_{\alpha, \beta}\right)
$$

is a spectral triple associated to the $C^{*}$-algebra $\mathcal{T}$. This extended spectral triple is s-summable if and only if the given one is s-summable.

Proof. Having the Lemma 1.9 we just have to prove that each $D_{\alpha, \beta}$ has compact resolvents, but that follows immediately from the definition of $D_{\alpha, \beta}$. Since $P$ commutes with the spectral projections for $D$, each eigenspace $H_{\lambda_{i}}$ for $D$ decomposes as an orthogonal sum $P H_{\lambda_{i}} \oplus(I-$ $P) H_{\lambda_{i}}$ and we can find an orthonormal basis for $P H$, say $\left(e_{i}\right)$, consisting of eigenvectors for $D_{p}$, plus an orthonormal basis for $(I-P) H$, say $\left(f_{j}\right)$, consisting of eigenvectors for $D_{q}$. If $e_{i}$ is an eigenvector corresponding to the eigenvalue $\lambda_{i}$, the operator $D_{\alpha, \beta}$ will have an invariant 2 dimensional subspace of the form $\left\{\left(z e_{i}, w e_{i}, 0\right) \mid z, w \in \mathbb{C}\right\}$ in the decomposition of $K$. The eigenvalues of $D_{\alpha, \beta}$ on this space are determined by the $2 \times 2$ matrix

$$
M(\alpha, \beta):=\left(\begin{array}{cc}
0 & \beta \\
\beta & \frac{1}{\alpha}
\end{array}\right)
$$

such that the eigenvalues become $\lambda_{i}$ times the eigenvalues of $M(\alpha, \beta)$. For an eigenvector $f_{j}$ for $D_{q}$ corresponding to an eigenvalue $\mu_{j}$ this vector becomes an eigenvector for $D_{\alpha, \beta}$ corresponding to the eigenvalue $\mu_{j} / \alpha$. Let now $s$ denote a positive real and we see that we get the equality below

$$
\operatorname{Tr}\left(\left|D_{\alpha, \beta}\right|^{-s}\right)=\operatorname{Tr}\left(|M(\alpha, \beta)|^{-s}\right) \operatorname{Tr}\left(\left|D_{p}\right|^{-s}\right)+\alpha^{s} \operatorname{Tr}\left(\left|D_{q}\right|^{-s}\right)
$$

and the proposition follows.

While we are at such matrix computations we remind you that for positive real numbers $\alpha, \beta, \gamma, \delta$, Hilbert spaces $L, M, N$ and bounded operators $v \in B(M, L), x \in B(L, M), y \in B(M), z \in B(N)$, we can obtain the identities below with respect to some operator matrices on Hilbert sum $L \oplus M \oplus N$. 


$$
\begin{aligned}
& \left(\begin{array}{ccc}
0 & \beta v & 0 \\
\beta x & \frac{1}{\alpha} y & 0 \\
0 & 0 & \frac{1}{\alpha} z
\end{array}\right)= \\
& (7) \\
& \left(\begin{array}{ccc}
\sqrt{\frac{\alpha}{\gamma}}\left(\frac{\beta}{\delta}\right) I & 0 & 0 \\
0 & \sqrt{\frac{\gamma}{\alpha}} I & 0 \\
0 & 0 & \sqrt{\frac{\gamma}{\alpha}} I
\end{array}\right)\left(\begin{array}{ccc}
0 & \delta v & 0 \\
\delta x & \frac{1}{\gamma} y & 0 \\
0 & 0 & \frac{1}{\gamma} z
\end{array}\right)\left(\begin{array}{ccc}
\sqrt{\frac{\alpha}{\gamma}}\left(\frac{\beta}{\delta}\right) I & 0 & 0 \\
0 & \sqrt{\frac{\gamma}{\alpha}} I & 0 \\
0 & 0 & \sqrt{\frac{\gamma}{\alpha}} I
\end{array}\right)
\end{aligned}
$$

and by (5) we can conclude as stated in the following lemma.

Lemma 1.12. Let a be in $A, k$ in $A_{c}$ and $\alpha, \beta, \gamma, \delta$ positive real numbers such that $\alpha \beta \leq 1$ and $\gamma \delta \leq 1$. For $t:=T(a)+k$ :

$$
\left\|\left[D_{\alpha, \beta}, \pi(t)\right]\right\| \leq \max \left\{\frac{\gamma}{\alpha}, \frac{\alpha \beta^{2}}{\gamma \delta^{2}}\right\}\left\|\left[D_{\gamma, \delta}, \pi(t)\right]\right\| .
$$

We will use this result heavily in the computations to come.

\section{The family of Compact quantum metric spaCes $\left(A_{\mathcal{T}}, L_{\alpha, \beta}\right)$}

For a spectral triple $(A, H, D)$ associated to a unital $\mathrm{C}^{*}$-algebra $\mathcal{A}$, Connes has showed that it is possible to define a metric on the state space $S(\mathcal{A})$ of $\mathcal{A}$ by the following formula

(8) $\forall \phi, \psi \in S(\mathcal{A}): \quad \operatorname{dist}_{\mathcal{A}}(\phi, \psi):=\sup \{|(\phi-\psi)(a)| \mid\|[D, a]\| \leq 1\}$.

A metric defined in this generality is allowed to be infinite, but here we are mostly interested in spectral triples which have the extra property that the metric defined above is an ordinary metric, which also is a metric for the $\mathrm{w}^{*}$-topology on the state space. This aspect of non commutative geometry has been studied in several articles by Marc Rieffel [26] and references there. Rieffel has generalized this set up to what he calls compact quantum metric spaces. Here the algebra $A$ of the spectral triple is replaced by an order unit space and the Dirac operator is not directly present, but replaced by a seminorm $L$ on $A$. In the case where a spectral triple is present the seminorm is given by $A \ni a \rightarrow L(a):=\|[D, a]\|$. Our investigation will not be so general here, since we will only study degenerations of spectral triples as constructed in the previous section. On the other hand we will base our results on Rieffel's memoir [27], and we will use the language from that memoir to quantify the impact of the changes of the parameters 
$\alpha$ and $\beta$. We will now recall some definitions and results from that memoir.

Definition 2.1. An order-unit space is a real partially ordered vector space, $A$, with a distinguished element e (the order unit) which satisfies:

(i) (Order unit property) For each $a \in A$ there is an $r \in \mathbb{R}$ such that $a \leq r e$.

(ii) (Archimedean property) If $a \in A$ and if $a \leq$ re for all $r \in \mathbb{R}$ with $r>0$, then $a \leq 0$.

The norm on an order-unit space is given by

$$
\|a\|=\inf \{r \in \mathbb{R}:-r e \leq a \leq r e\} .
$$

Any order-unit space can be realized as a real linear subspace of the vector space of self-adjoint bounded operators on a Hilbert space in such a way that the order unit is the unit operator $I$.

Definition 2.2. Let $(A, e)$ be an order-unit space, and its dual, $A^{*}$. The state space $S(A)$ is defined to be the collection of all states, $\mu$, of A, i.e. $\mu \in A^{*}$ such that $\mu(e)=1=\|\mu\|$.

Consider now a seminorm $L$ on the order-unit space $(A, e)$ having its null-space equal to the scalar multiples of the order unit. Then, for $\mu, \nu \in S(A)$ one can define a metric, $\rho_{L}$, on $S(A)$ by

$$
\rho_{L}(\mu, \nu):=\sup \{|\mu(a)-\nu(a)| \mid L(a) \leq 1\} .
$$

In absence of further assumptions, $\rho_{L}(\mu, \nu)$ may be infinite. It is most often true that the $\rho$-topology on $S(A)$ is finer than the weak*-topology.

Definition 2.3. Let $(A, e)$ be an order-unit space. A Lip-norm on $A$ is a seminorm $L$ on $A$ with the following properties:

(i) For $a \in A$ we have $L(a)=0$ if and only if $a \in \mathbb{R} e$.

(ii) The topology on $S(A)$ from the metric $\rho_{L}$ is the weak*-topology.

Definition 2.4. A compact quantum metric space is a pair $(A, L)$ consisting of an order-unit space $A$ with a Lip-norm $L$ defined on it.

In our context we have four $\mathrm{C}^{*}$-algebras $C(P H), \mathcal{A}, \mathcal{T}$, and the unitarization of the compacts which we define by $\mathcal{C}:=\widehat{C(P H)}:=$ $C(P H)+\mathbb{C} I_{P H}$. We will now define the order unit spaces and associated Lip-norms, which we will study.

Definition 2.5. The order unit spaces $A_{\mathcal{C}}, A_{\mathcal{A}}, A_{\mathcal{T}}$ are defined by:

$$
\begin{aligned}
& A_{\mathcal{C}}:=\left\{k+\lambda I \mid \lambda \in \mathbb{R}, k=k^{*} \in A_{c}\right\} \text { Definition } 1.8 \\
& A_{\mathcal{A}}:=\left\{a \in A \mid a=a^{*}\right\} \\
& A_{\mathcal{T}}:=T\left(A_{\mathcal{A}}\right)+A_{\mathcal{C}}=\left\{t \in A_{t} \mid t=t^{*}\right\}
\end{aligned}
$$


Definition 2.6. The seminorms $L_{\mathcal{C}}, L_{\mathcal{A}}$ and $L_{\alpha, \beta}$ on $A_{\mathcal{C}}, A_{\mathcal{A}}$ and $A_{\mathcal{T}}$ are defined by

$$
\begin{aligned}
& \forall k \in A_{\mathcal{C}} \cap C(P H) \forall \lambda \in \mathbb{R}: L_{\mathcal{C}}(k+\lambda I):=\left\|D_{p} k\right\| . \\
& \forall a \in A_{\mathcal{A}}: L_{\mathcal{A}}(a):=\|[D, a]\| \\
& \forall t \in A_{\mathcal{T}}: L_{\alpha, \beta}(t):=\left\|\left[D_{\alpha, \beta}, \pi(t)\right]\right\| .
\end{aligned}
$$

The corresponding Minkowski sets or unit balls are defined by

\section{Definition 2.7.}

$$
\begin{aligned}
U_{\mathcal{C}} & :=\left\{x \in A_{\mathcal{C}} \mid L_{\mathcal{C}}(x) \leq 1\right\} \\
U_{\mathcal{A}} & :=\left\{a \in A_{\mathcal{A}} \mid L_{\mathcal{A}}(a) \leq 1\right\} \\
U_{\alpha, \beta} & :=\left\{t \in A_{\mathcal{T}} \mid L_{(\alpha, \beta)}(t) \leq 1\right\}
\end{aligned}
$$

The associated metrics are given by

\section{Definition 2.8.}

$$
\begin{aligned}
\forall f, g \in S(\widetilde{C(P H})): \operatorname{dist}_{\mathcal{C}}(f, g) & :=\sup \left\{|f(k)-g(k)| \mid k \in U_{\mathcal{C}}\right\} \\
(20) \quad \forall \mu, \nu \in S(\mathcal{A}): \operatorname{dist}_{\mathcal{A}}(\mu, \nu) & :=\sup \left\{|\mu(a)-\nu(a)| \mid a \in U_{\mathcal{A}}\right\} \\
(21) \quad \forall \phi, \psi \in S(\mathcal{T}): \operatorname{dist}_{\alpha, \beta}(\phi, \psi) & :=\sup \left\{|\phi(t)-\psi(t)| \mid t \in U_{(\alpha, \beta)}\right\} .
\end{aligned}
$$

The diameters of these spaces, which may take the value $\infty$, are denoted $\operatorname{diam}_{C}, \operatorname{diam}_{\mathcal{A}}$ and $\operatorname{diam}_{\alpha, \beta}$ respectively.

Let $X$ denote any of the 3 subscripts $\mathcal{C}, \mathcal{A},(\alpha, \beta)$, then it follows from [28] that the metric dist ${ }_{X}$ defined above is a metric for the $\mathrm{w}^{*}$ topology on the corresponding state space, if and only if the set $U_{X}$ is separating for the state space, and the image of $U_{X}$ in the quotient space $A_{X} /(\mathbb{R} I)$ is relatively norm compact $\left(A_{\alpha, \beta}:=A_{\mathcal{T}}\right)$. We start by showing that $L_{\mathcal{C}}$ is always a Lip-norm and then we show that any $L_{\alpha, \beta}$ is a Lip-norm if $L_{\mathcal{A}}$ is so.

Proposition 2.9. The seminorm $L_{\mathcal{C}}$ is a Lip-norm.

Proof. Let us first prove that $U_{\mathcal{C}}$ will separate the state space of $\widetilde{C(P H)}$. To this end we remind you that the spectrum of $D_{p}$ is discrete and $D_{p}$ has a compact inverse (on $P H$ ), since it has a trivial kernel. Let then $h:=D_{p}^{-1}$ and it follows that the set $\left\{h y h \mid y \in B(P H)\right.$ and $\left.y=y^{*}\right\}$ is contained in $A_{\mathcal{C}} \cap C(P H)$. Since $h$ is selfadjoint with trivial kernel ( in $B(P H))$ this set is norm dense in the self-adjoint part of $C(P H)$, and it follows that the set $\left\{h y h \mid y \in B(P H)\right.$ and $\left.y=y^{*}\right\}$ separates the states and that $U_{\mathcal{C}}$ will do too. 
Let us define $U_{\mathcal{C}}^{\circ}:=\left\{k \in A_{\mathcal{C}} \cap C(P H) \mid\left\|D_{p} k\right\| \leq 1\right\}$, and we will prove that this set is norm compact. From here it will follow directly that $U_{\mathcal{C}} /(\mathbb{R} I)=\left(U_{\mathcal{C}}^{\circ}+\mathbb{R} I\right) /(\mathbb{R} I)$ is norm compact.

To see that $U_{\mathcal{C}}^{\circ}$ is norm compact we will let $\varepsilon$ denote a positive real and recall that $h$ is compact, so there exists a finite dimensional spectral projection $E$ for $h$ such that $\|h(I-E)\|<\varepsilon$. For a $k$ in $U_{\mathcal{C}}^{\circ}$ we then have

$$
\|k(I-E)\|=\left\|k D_{p} h(I-E)\right\| \leq \varepsilon\left\|k D_{p}\right\| \leq \varepsilon .
$$

and since $k$ is self-adjoint

$$
\|(I-E) k\| \leq \varepsilon \text {. }
$$

Then for the set $U_{\mathcal{C}}^{\circ}$ we get $U_{\mathcal{C}}^{\circ}=U_{\mathcal{C}}^{\circ}(I-E)+(I-E) U_{\mathcal{C}}^{\circ} E+E U_{\mathcal{C}}^{\circ} E$, where each operator in either of the first two summands is of norm at most $\varepsilon$ and the set $E U_{\mathcal{C}}^{\circ} E$ is the unit ball for some norm on the finite dimensional space $B(E H)$. It then follows that $U_{\mathcal{C}}^{\circ}$ is relatively norm compact. To see that it is norm closed, we consider a sequence $\left(k_{n}\right)$ of elements from $U_{\mathcal{C}}^{\circ}$ which converges in norm to a compact self-adjoint operator $k$. For any spectral projection $E$ of $D_{p}$ corresponding to a bounded interval of the real numbers we have that the sequence $\left(D_{p} E k_{n}\right)$ is norm convergent with limit $D_{p} E k$, and we see that $\left\|D_{p} E k\right\| \leq 1$, and therefore $k$ belongs to $U_{\mathcal{C}}^{\circ}$.

We will end this section by showing that each of the seminorms $L_{\alpha, \beta}$ is a Lip-norm if the seminorm $L_{\mathcal{A}}$ is a Lip-norm. This leads to a detailed study - in the next section - of the two parameter family of compact quantum metric spaces, $\left(A_{\mathcal{T}}, L_{\alpha, \beta}\right)$. On the other hand we already now need some estimates on the relations between the various seminorms in order to prove that dist $_{\alpha, \beta}$ generates the $\mathrm{w}^{*}$-topology, if $\operatorname{dist}_{\mathcal{A}}$ does so.

Lemma 2.10. Let $\alpha, \beta$ be positive reals such that $\alpha \beta \leq 1, k$ a compact operator in $A_{\mathcal{C}}$ and a an operator in $A_{\mathcal{A}}$ then $t=k+T(a)$ is in $A_{\mathcal{T}}$ and

$$
\begin{aligned}
L_{\mathcal{A}}(a) & \leq \alpha L_{\alpha, \beta}(t) \\
L_{\mathcal{C}}(k) & \leq \frac{1+\alpha \beta}{\beta} L_{\alpha, \beta}(t)
\end{aligned}
$$

Proof. The first inequality follows directly from (5) and properties of norms of matrices. For the second inequality we use again (5), the 
result in the first inequality and the triangle inequality to obtain

$$
\begin{aligned}
\beta L_{\mathcal{C}}(k) & \leq L_{\alpha, \beta}(t)+\beta\left\|\left[D_{p}, T(a)\right]\right\| \leq L_{\alpha, \beta}(t)+\beta L_{\mathcal{A}}(a) \\
& \leq(1+\alpha \beta) L_{\alpha, \beta}(t) .
\end{aligned}
$$

and the lemma follows.

Proposition 2.11. If $L_{\mathcal{A}}$ is a Lip-norm, then for each pair of positive reals $(\alpha, \beta)$ such that $\alpha \beta \leq 1$ the seminorm $L_{\alpha, \beta}$ is a Lip-norm.

Proof. To see that $U_{\alpha, \beta} /(\mathbb{R} I)$ is relatively norm compact we turn back to Lemma 2.10, which implies that for a $t=T(a)+k$ in $U_{\alpha, \beta}$ we have that

$$
a \in \alpha U_{\mathcal{A}} \text { and } k \in \frac{1+\alpha \beta}{\beta} U_{C}
$$

So

$$
U_{\alpha, \beta} \subseteq \alpha T\left(U_{\mathcal{A}}\right)+\frac{1+\alpha \beta}{\beta} U_{C}
$$

and

$$
U_{\alpha, \beta} /(\mathbb{R} I) \subseteq \alpha T\left(U_{\mathcal{A}} /(\mathbb{R} I)\right)+\frac{1+\alpha \beta}{\beta} U_{C} /(\mathbb{R} I) .
$$

Since $\operatorname{dist}_{\mathcal{A}}$ generates a metric for the $\mathrm{w}^{*}$-topology on $S(\mathcal{A})$ the set $U_{\mathcal{A}} /(\mathbb{R} I)$ is relatively norm compact in $A_{\mathcal{A}} /(\mathbb{R} I)$, and from the proof of Proposition 2.9 we know that that $U_{\mathcal{C}} /(\mathbb{R} I)$ is a norm compact subset of $A_{\mathcal{C}} /(\mathbb{R} I)$ so we find that $U_{\alpha, \beta} /(\mathbb{R} I)$ is a relatively norm compact subset of $A_{\mathcal{T}} /(\mathbb{R} I)$.

In the recent article [26] Rieffel studies Lip-norms which satisfy some extra conditions, which he needs in order to show certain results on convergence in the space of compact quantum metric spaces, equipped with the quantum Gromov-Hausdorff metric. The new seminorms are called $C^{*}$-seminorms and it seems most likely that the seminorms we study may possess most of the properties which a $\mathrm{C}^{*}$-seminorm is required to have. We will not recall all of the definitions from [26], but just recall that one of the properties is that such a seminorm is demanded to be lower semicontinuous. In our context this means that the set $\left\{t \in A_{\mathcal{T}} \mid\left\|L_{\alpha, \beta}(t)\right\| \leq 1\right\}$ is norm closed. It seems quite unlikely to be the case here since we have imposed some regularity conditions in Definition 1.2. on the set $A_{\mathcal{A}}$. This means that already the seminorm $L_{\mathcal{A}}$ will probably not in general be lower semicontinuous. On the other hand we might extend such a seminorm to a larger subalgebra of $\mathcal{T}$ and in this way obtain a lower semicontinuous seminorm, but then it seems difficult for an operator $t$ in the extended domain for $L_{\alpha, \beta}$ to 
control the behavior of the matrix matrix parts of the commutators of the form $\left[D_{\alpha, \beta}, t\right]$.

We are very thankful to Hanfeng Li, who has showed us, how it is possible to prove that the seminorm $L_{\alpha, \beta}$ has the two other properties of a $\mathrm{C}^{*}$-seminorm named spectral stability and strongly Leibniz, provided the original seminorm $L_{\mathcal{A}}$ has these properties. On the other hand it seems that the regularity conditions, we have imposed, may be in conflict with the possibility for $L_{\mathcal{A}}$ to be spectrally stable. Mainly inspired by the classical case we have the impression that the difficluties of this type may be avoided if we restrict our construction to the special case, where the domain of definitions for the seminorms, $A_{\mathcal{A}}$ and $A_{\mathcal{T}}$ are only the smooth elements as defined by Connes in his smoothness axiom of [8]. We will present and discuss this axiom in Section 6 .

\section{The COMPaCt QuAntum metric SPACES ASSOCiated to $\mathcal{T}$}

In this section we will suppose that the seminorm $L_{\mathcal{A}}$ is a Lip-norm, and then by Proposition 2.11 all the tuples $\left(A_{\mathcal{T}}, L_{\alpha, \beta}\right)$ are compact quantum metric spaces. This means that the metric spaces

$$
\left\{\left(S(\mathcal{T}), \operatorname{dist}_{\alpha, \beta}\right) \mid 0<\alpha \beta \leq 1\right\}
$$

are equipped with the $\mathrm{w}^{*}$-topology and hence they are ordinary compact metric spaces. It seems natural to compare these metric spaces by obtaining Lipschitz estimates between any pair of two metrics. Based on the Lemma 1.12 we can quite easily obtain such results, which we present just below. The spaces we are studying are not only compact metric spaces but also compact quantum metric spaces and Rieffel has in the memoir [27] developed a distance concept for such spaces called the quantum Gromov-Hausdorff distance. This last concept of distance is based on the Hausdorff metric on the closed subsets of a compact metric space. Gromov has extended this idea and introduced a distance function defined on pairs of compact metric spaces, and finally Rieffel [27] has extended Gromov's ideas to cover the case of compact quantum metric spaces. We will return to this definition shortly, but first we will treat the Lipschitz estimates between a pair of metrics $\operatorname{dist}_{\alpha, \beta}$ and $\operatorname{dist}_{(\gamma, \delta)}$ on $S(\mathcal{T})$.

Proposition 3.1. For any positive reals $\alpha, \beta, \gamma, \delta$ such that $\alpha \beta \leq 1$, $\gamma \delta \leq 1$ and any $t$ in $A_{\mathcal{T}}$

$$
\min \left\{\frac{\gamma}{\alpha}, \frac{\alpha \beta^{2}}{\gamma \delta^{2}}\right\} L_{\gamma, \delta}(t) \leq L_{\alpha, \beta}(t) \leq \max \left\{\frac{\gamma}{\alpha}, \frac{\alpha \beta^{2}}{\gamma \delta^{2}}\right\} L_{\gamma, \delta}(t)
$$


Proof. We will only prove the right inequality, since the left then follows by symmetry. As usual we have a decomposition of $t$ in $A_{\mathcal{T}}$ as the sum $T(a)+k$ with $a$ in $A_{\mathcal{A}}$ and $k$ in $A_{\mathcal{C}} \cap C(P H)$. When going back to the definition in (14) we get

$$
L_{\alpha, \beta}(t)=\left\|\left[D_{\alpha, \beta}, \pi(t)\right]\right\|
$$

and from the results of Lemma 1.12 we then get

$$
L_{\alpha, \beta}(t) \leq \max \left\{\frac{\gamma}{\alpha}, \frac{\alpha \beta^{2}}{\gamma \delta^{2}}\right\} L_{\gamma, \delta}(t) .
$$

The results of Proposition 3.1 may be applied to the metrics $\operatorname{dist}_{\alpha, \beta}$ and we can obtain the following proposition.

Theorem 3.2. Let $\alpha, \beta, \gamma, \delta$ be positive reals such that $\alpha \beta \leq 1$ and $\delta \gamma \leq 1$, then the metrics $\operatorname{dist}_{\alpha, \beta}(\cdot, \cdot)$ and $\operatorname{dist}_{\gamma, \delta}(\cdot, \cdot)$ on $S(\mathcal{T})$ are Lipschitz equivalent and satisfy the following inequalities

$$
\begin{aligned}
\forall \phi, \psi \in S(\mathcal{T}): & \min \left\{\frac{\gamma}{\alpha}, \frac{\alpha \beta^{2}}{\gamma \delta^{2}}\right\} \operatorname{dist}_{\alpha, \beta}(\phi, \psi) \leq \operatorname{dist}_{\gamma, \delta}(\phi, \psi) \\
& \leq \max \left\{\frac{\gamma}{\alpha}, \frac{\alpha \beta^{2}}{\gamma \delta^{2}}\right\} \operatorname{dist}_{\alpha, \beta}(\phi, \psi) .
\end{aligned}
$$

Proof. The Proposition 3.1 shows that with the notation from Definition 2.7 we get

$$
\min \left\{\frac{\gamma}{\alpha}, \frac{\alpha \beta^{2}}{\gamma \delta^{2}}\right\} U_{\alpha, \beta} \subseteq U_{\gamma, \delta} \subseteq \max \left\{\frac{\gamma}{\alpha}, \frac{\alpha \beta^{2}}{\gamma \delta^{2}}\right\} U_{\alpha, \beta} .
$$

The theorem then follows from the definition given in (21).

We have now seen that any two metrics in this two parameter family of metrics on $S(\mathcal{T})$ are Lipschitz equivalent, and it follows from this that we can deduce estimates of the distance with respect to a quantum Gromov-Hausdorff metric between the compact quantum metric spaces $\left(A_{\mathcal{T}}, L_{\alpha, \beta}\right)$ and $\left(A_{\mathcal{T}}, L_{\gamma, \delta}\right)$.

We shall first review, briefly, the Gromov-Hausdorff distance for compact metric spaces and Rieffel's quantum distance for compact quantum metric spaces. We use as references [22] and [27]. For any closed subset $Y$ of a metric space $(X, \rho)$ and $r>0$, we denote:

$$
\mathcal{N}_{r}^{\rho}(Y):=\{x \in X: \exists y \in Y \text { with } \rho(x, y) \leq r\} .
$$


Let $\mathcal{S}$ denote the class of all non-empty closed subsets of $X$. The formula,

$\forall Y, Z \in \mathcal{S}: \operatorname{dist}_{\mathrm{H}}^{\rho}(Y, Z):=\inf \left\{r: Y \subseteq \mathcal{N}_{r}^{\rho}(Z)\right.$ and $\left.Z \subseteq \mathcal{N}_{r}^{\rho}(Y)\right\}$,

defines a metric (called the Hausdorff metric) on $\mathcal{S}$. One can also use the notation $\operatorname{dist}_{\mathrm{H}}^{X}(Y, Z)$ when there is no confusion about the metric on $X$.

Gromov generalized the Hausdorff distance to a distance between any two compact metric spaces $X, Y$ as follows

$$
\operatorname{dist}_{\mathrm{GH}}(X, Y):=\inf \left\{\operatorname{dist}_{\mathrm{H}}^{Z}\left(h_{X}(X), h_{Y}(Y)\right) \mid h_{X}: X \rightarrow Z, h_{Y}: Y \rightarrow Z\right.
$$

are isometric embeddings into some compact metric space $Z$ \}.

One can reduce the space $Z$ above to be the disjoint union $X \amalg Y$, and we shall denote with $\mathcal{D}(X, Y)$ the set of all distances $\rho$ on $X \amalg Y$ fulfilling that the inclusions $X, Y \hookrightarrow X \amalg Y$ are isometric embeddings. It is then true that

$$
\operatorname{dist}_{\mathrm{GH}}(X, Y):=\inf \left\{\operatorname{dist}_{\mathrm{H}}^{\rho}(X, Y): \rho \in \mathcal{D}(X, Y)\right\} .
$$

Let $A$ be an order-unit space. By a quotient $(B, \pi)$ of $A$, we mean an order-unit space $B$ and a surjective linear positive map $\pi: A \rightarrow$ $B$ preserving the order-unit. Via the dual map $\pi^{*}: B^{*} \rightarrow A^{*}$, one may identify $S(B)$ with a closed convex subset of $S(A)$. This gives a bijection between isomorphism classes of quotients of $A$ and closed convex subsets of $S(A)$. If $L$ is a Lip-norm on $A$, then the quotient seminorm $L_{B}$ on $B$, defined by

$$
L_{B}(b):=\inf \{L(a): \pi(a)=b\}
$$

is a Lip-norm on $B$, and $\left.\pi^{*}\right|_{S(B)}: S(B) \rightarrow S(A)$ is an isometry for the corresponding metrics $\rho_{L}$ and $\rho_{L_{B}}$.

Let $\left(A, L_{A}\right)$ and $\left(B, L_{B}\right)$ be compact quantum metric spaces. The direct sum $A \oplus B$ has naturally the structure of an order unit space with order unit $\left(e_{A}, e_{B}\right)$. We will let $\mathcal{M}\left(L_{A}, L_{B}\right)$ denote the the set of all Lipnorms $L$ on $A \oplus B$ that induces $L_{A}$ and $L_{B}$ under the natural quotient maps $A \oplus B \mapsto A$ and $A \oplus B \mapsto B$. For an element $L$ in $\mathcal{M}\left(L_{A}, L_{B}\right)$ with the associated metric $\rho_{L}$ on $S(A \oplus B)$, it is then possible to consider both of the compact metric spaces $\left(S(A), \rho_{L_{A}}\right)$ and $\left(S(B), \rho_{L_{B}}\right)$ as compact subsets of the compact metric space $\left(S(A \oplus B), \rho_{L}\right)$, and one can then compute the usual Hausdorff distance between them. This distance is denoted dist $_{H}^{\rho_{L}}(S(A), S(B))$. We can then define a metric on compact quantum metric spaces as follows. 
Definition 3.3. Let $\left(A, L_{A}\right)$ and $\left(B, L_{B}\right)$ be compact quantum metric spaces. Then the quantum Gromov-Hausdorff distance between them is denoted

$\operatorname{dist}_{q}(A, B)$ and it is defined by

$$
\operatorname{dist}_{q}\left(\left(A, L_{A}\right),\left(B, L_{B}\right)\right):=\inf \left\{\operatorname{dist}_{H}^{\rho_{L}}(S(\mathcal{A}), S(\mathcal{B})) \mid L \in \mathcal{M}\left(L_{A}, L_{B}\right)\right\}
$$

Li gave in [22] the following description of the Gromov-Hausdorff distance.

Proposition 3.4. Let $\left(A, L_{A}\right)$ and $\left(B, L_{B}\right)$ be compact quantum metric spaces. Then we have

$$
\begin{aligned}
\operatorname{dist}_{q}\left(\left(A, L_{A}\right),\left(B, L_{B}\right)\right)= & \inf \left\{\operatorname{dist}_{H}^{V}\left(h_{A}(S(A)), h_{B}(S(B))\right):\right. \\
& h_{A}, h_{B} \text { are affine isometric embeddings of } \\
& S(A), S(B) \text { into some real normed space } V\} .
\end{aligned}
$$

This tells us that the quantum Gromov-Hausdorff distance between two compact quantum metric spaces $\left(A, L_{A}\right)$ and $\left(B, L_{B}\right)$ always will be larger or equal to the Gromov-Hausdorff distance between the compact metric spaces $\left(S(A), \rho_{L_{A}}\right)$ and $\left(S(B), \rho_{L_{B}}\right)$.

Besides Rieffel and Li, there are by now several mathematicians who have published articles on convergence and estimates of distances between compact quantum metric spaces and even incorporated the extra structure coming from the theory of operator spaces into their research [17], [21], [30] and we have found this very stimulating for the present work.

We will now use the results of Proposition 3.1 to compute estimates for the distance between a pair $\left(A_{\mathcal{T}}, L_{\alpha, \beta}\right)$ and $\left(A_{\mathcal{T}}, L_{\gamma, \delta}\right)$ of compact quantum metric spaces. Our construction is based on Rieffel's concept called a bridge, but we could not get his concept to fit exactly into our frame, so we have modified it a bit and incorporated the idea of a bridge into the proof of the following proposition. On the other hand our situation is much simpler than the general situation, considered by Rieffel, since the order unit space is kept fixed as $A_{\mathcal{T}}$.

Proposition 3.5. Let $A$ be an order unit space and let $L_{1}$ and $L_{2}$ be two Lip-norms on A for which there exist positive real number $s<r$ such that

$$
\forall a \in A: \quad s L_{2}(a) \leq L_{1}(a) \leq r L_{2}(a)
$$


Define $L_{3}:=(1 / \sqrt{r s}) L_{1}$, let dist $_{3}$, dist ${ }_{2}$ be the metrics induced by $L_{3}, L_{2}$ and let $\operatorname{diam}_{3}, \operatorname{diam}_{2}$ denote the diameters of the compact metric spaces $\left(S(A)\right.$, dist $\left._{3}\right),\left(S(A)\right.$, dist $\left._{2}\right)$ then

$$
\operatorname{dist}_{q}\left(\left(A, L_{3}\right),\left(A, L_{2}\right)\right) \leq\left(\sqrt{\frac{r}{s}}-1\right) \min \left\{\operatorname{diam}_{3}, \operatorname{diam}_{2}\right\} .
$$

Proof. We first fix an arbitrary base point, which in this case means a state $\sigma$ on $A$, and then we let $M$ denote an arbitrary positive real. Later in the argument we will let $M$ increase unlimited, so you may think of $M$ as a big positive real. We will let $R$ denote the positive real which is defined by

$$
R:=\frac{\sqrt{s}}{\sqrt{r}-\sqrt{s}}
$$

and we can then define a seminorm $L$ on $A \oplus A$ by

$\forall a, b \in A:$

$L(a, b):=\max \left\{L_{3}(a), L_{2}(b), R L_{3}(a-b), R L_{2}(a-b), M|\sigma(a-b)|\right\}$.

Since $L$ is defined as a maximum over seminorms, it follows that $L$ is a seminorm on $A \oplus A$. If $L(a, b)=0$ then since $L_{3}$ and $L_{2}$ are Lipnorms we see that $a=\alpha I$ and $b=\beta I$ for some real numbers $\alpha, \beta$ and finally $\sigma(a-b)=0$ implies that $\alpha=\beta$, so $(a, b)=\alpha(I, I)$ and the first condition for $L$ being a Lip-norm is established. We will of course also show that $L$ belongs to $\mathcal{M}\left(L_{3}, L_{2}\right)$, and we will address the question of whether $L$ induces $L_{3}$ and $L_{2}$ on the summands first. Let us start by looking at the first summand and $L_{3}$ first. We then define the following sets.

$$
\begin{aligned}
U_{L} & :=\{(a, b) \in A \oplus A \mid L(a, b) \leq 1\} \\
U_{L \mid A} & :=\left\{a \in A \mid \exists b \in A:(a, b) \in U_{L}\right\} \\
U_{2} & :=\left\{b \in A \mid L_{2}(b) \leq 1\right\} \\
U_{3} & :=\left\{a \in A \mid L_{3}(a) \leq 1\right\}
\end{aligned}
$$

In order to prove that $L$ induces $L_{3}$ it is sufficient to prove that $U_{L \mid A}=$ $U_{3}$, so we will do that. By definition $L(a, b) \geq L_{3}(a)$ so for any pair $(a, b) \in U_{L}$ we have $a \in U_{3}$, and then $U_{L \mid A} \subseteq U_{3}$. To establish the opposite inclusion we choose an $a \in U_{3}$ and construct a suitable $b$ such that $(a, b)$ is in $U_{L}$. It is a matter of checking to show that the element $b$ in $A$ defined by $b:=\sqrt{s / r} a+(1-\sqrt{s / r}) \sigma(a) I$ will do. The situation for the second summand is very similar, and it turns 
out that for any $b$ in $A$ such that $L_{2}(b) \leq 1$ we can define $a$ in $A$ by $a:=\sqrt{s / r} b+(1-\sqrt{s / r}) \sigma(b) I$, and then $L(a, b) \leq 1$.

The seminorm $L$ is defined on all of $A \oplus A$ so the set $U_{L}$ will be separating for the states on $A \oplus A$.

We then just have to prove that the set $U_{L} /(\mathbb{R}(I, I))$ is relatively norm compact in the quotient space $(A \oplus A) /(\mathbb{R}(I, I))$. Let $\tilde{\sigma}$ denote the state on $A \oplus A$ given by $\tilde{\sigma}(a, b):=\sigma(a)$, then it is standard to deduce that $U_{L} /(\mathbb{R}(I, I))$ is relatively norm compact if and only if the set $U_{\sigma}:=\{(a, b) \in U \mid \tilde{\sigma}(a, b)=0\}$ is relatively norm compact in $A \oplus A$. This implies that we may define two relatively norm compact sets in $A$ by $U_{(3, \sigma)}:=\left\{a \in U_{3} \mid \sigma(a)=0\right\}$ and $U_{(2, \sigma, M)}:=\left\{b \in U_{2}|| \sigma(b) \mid \leq M\right\}$. For these sets we find that $U_{\sigma} \subseteq U_{(3, \sigma)} \oplus U_{(2, \sigma, M)}$ so the metric $\rho_{L}$ generates the w*-topology on $S(A \oplus A)$.

We can now use this metric to get an upper estimate for the quantum Gromov-Hausdorff distance and we find that for any state $\phi$ on $A$

$$
\begin{aligned}
& \rho_{L}((\phi, 0),(0, \phi))=\sup \left\{|\phi(a-b)| \mid(a, b) \in U_{L}\right\} \\
& \leq \sup \left\{|(\phi-\sigma)(a-b)| \mid(a, b) \in U_{L}\right\}+\frac{1}{M} \\
& \leq \min \left\{\frac{\operatorname{diam}_{3}}{R}, \frac{\operatorname{diam}_{2}}{R}\right\}+\frac{1}{M}, \text { since }(a-b) \in \frac{1}{R} U_{3} \cap \frac{1}{R} U_{2}
\end{aligned}
$$

By letting $M$ grow we conclude that

$$
\operatorname{dist}_{q}\left(\left(A, L_{3}\right),\left(A, L_{2}\right)\right) \leq(\sqrt{r / s}-1) \min \left\{\operatorname{diam}_{3}, \operatorname{diam}_{2}\right\} .
$$

and the proposition follows.

Remark 3.6. In connection with the proposition above it may be relevant to note that the diameters diam $_{2}$, diam 3 relate in a reciprocal way as the corresponding seminorms, so we have

$$
\sqrt{s / r} \cdot \operatorname{diam}_{3} \leq \operatorname{diam}_{2} \leq \sqrt{r / s} \cdot \operatorname{diam}_{3} .
$$

The special case where the seminorms $L_{1}$ and $L_{2}$ are proportional is taken out as a corollary.

Corollary 3.7. Let $A$ be an order unit space with a Lip-norm L. For any positive real $t$ :

$$
\operatorname{dist}_{q}((A, L),(A, t L)) \leq|1-1 / t| \operatorname{diam}_{(A, L)} .
$$

Proof. Suppose $t>1$ then for the Lip-norm $N:=t^{2} L$ we have $L \leq$ $N \leq t^{2} L$. The proposition then applies with $s=1$ and $r=t^{2}$, so 
for $t L=(1 / \sqrt{s r}) N$ we get by the Remark 3.6 and the use of the $\min$ option in Proposition 3.5

$$
\begin{aligned}
\operatorname{dist}_{q}((A, L),(A, t L)) & \leq(\sqrt{r / s}-1) \operatorname{diam}_{t L} \\
& =(t-1) \operatorname{diam}_{t L} \\
& =\left(1-t^{-1}\right) \operatorname{diam}_{L} .
\end{aligned}
$$

For $t<1$ and $N=t^{2} L$ we get $t^{2} L \leq N \leq L$ and then

$$
\operatorname{dist}_{q}((A, L),(A, t L)) \leq\left(t^{-1}-1\right) \operatorname{diam}_{L} .
$$

The corollary above suggests that it could be interesting to see what will happen for $t$ increasing to infinity, so we will include such a result.

Proposition 3.8. Let $(A, L)$ be an order unit space with a Lip-norm, and let $(\mathbb{R}, 0)$ be the one point order unit space with Lip-norm equal to 0 . For any positive real $t$ we have the estimate.

$$
\operatorname{dist}_{q}((A, t L),(\mathbb{R}, 0)) \leq \frac{\operatorname{diam}_{(A, L)}}{t} .
$$

Proof. We choose and fix a state $\sigma$ on $A$ and let $M$ denote a big positive real. We can then define a seminorm $\hat{L}_{t}$ on $A \oplus \mathbb{R}$ by

$$
\hat{L}_{t}(a, s):=\max \{t L(a), M|\sigma(a)-s|\}
$$

It is easy to check that $\hat{L}_{t}$ induces the seminorms $t L$ on $A$ and the zero seminorm on $\mathbb{R}$. The order unit space $\mathbb{R}$ has exactly one state which we denote by $\psi$. For a state $\phi$ on $A$ we can estimate as follows.

$$
\begin{aligned}
& \operatorname{dist}_{\hat{L}_{t}}((\phi, 0),(0, \psi)) \\
& =\sup \left\{|\phi(a)-s| \mid \hat{L}_{t}(a, s) \leq 1\right\} \\
& \leq \sup \{|\phi(a)-\sigma(a)| \mid t L(a) \leq 1\}+\sup \left\{|\sigma(a)-s||| \sigma(a)-s \mid \leq \frac{1}{M}\right\} \\
& \leq \frac{\operatorname{diam}_{(A, L)}}{t}+\frac{1}{M}
\end{aligned}
$$

The proposition follows.

We can then combine some of the results just obtained with Proposition 3.1 to obtain estimates on the variation of the compact quantum metric spaces $\left(A_{\mathcal{T}}, L_{\alpha, \beta}\right)$. In this connection we will let $\operatorname{diam}_{\alpha, \beta}$ denote the diameter of this space. 
Theorem 3.9. If $\alpha, \beta, \delta, \gamma$ are positive reals such that $\alpha \beta \leq 1$ and $\gamma \delta \leq 1$ then:

$$
\begin{aligned}
& \operatorname{dist}_{q}\left(\left(A_{\mathcal{T}}, L_{\alpha, \beta}\right),\left(A_{\mathcal{T}}, L_{\gamma, \delta}\right)\right) \\
& \leq\left(\max \left\{\frac{\alpha \beta}{\gamma \delta}, \frac{\gamma \delta}{\alpha \beta}\right\}-1+\left|1-\frac{\beta}{\delta}\right|\right) \operatorname{diam}_{\alpha, \beta},
\end{aligned}
$$

Proof. Inspired by Proposition 3.1 we define

$$
s:=\min \left\{\frac{\gamma}{\alpha}, \frac{\alpha \beta^{2}}{\gamma \delta^{2}}\right\} \quad r:=\max \left\{\frac{\gamma}{\alpha}, \frac{\alpha \beta^{2}}{\gamma \delta^{2}}\right\},
$$

then we get

$$
\forall t \in A_{\mathcal{T}}: \quad s L_{\gamma, \delta}(t) \leq L_{\alpha, \beta}(t) \leq r L_{\gamma, \delta}(t) .
$$

In the notation from Proposition 3.5

$$
\frac{1}{\sqrt{r s}}=\frac{\delta}{\beta} \text { and } \sqrt{\frac{r}{s}}=\max \left\{\frac{\alpha \beta}{\gamma \delta}, \frac{\gamma \beta}{\alpha \delta}\right\},
$$

so we have the estimate

$$
\begin{aligned}
& \operatorname{dist}_{q}\left(\left(A_{\mathcal{T}}, L_{\gamma, \delta}\right),\left(A_{\mathcal{T}},(\delta / \beta) L_{\alpha, \beta}\right)\right) \\
& \leq\left(\max \left\{\frac{\alpha \beta}{\gamma \delta}, \frac{\gamma \delta}{\alpha \beta}\right\}-1\right) \operatorname{diam}_{\alpha, \beta} .
\end{aligned}
$$

We can then use Corollary 3.7 and the triangle inequality to get

$$
\begin{aligned}
& \operatorname{dist}_{q}\left(\left(A_{\mathcal{T}}, L_{\gamma, \delta}\right),\left(A_{\mathcal{T}}, L_{\alpha, \beta}\right)\right) \\
& \leq\left(\max \left\{\frac{\alpha \beta}{\gamma \delta}, \frac{\gamma \delta}{\alpha \beta}\right\}-1+\left|1-\frac{\beta}{\delta}\right|\right) \operatorname{diam}_{\alpha, \beta},
\end{aligned}
$$

and the theorem follows.

\section{ON Limits OF $\left(A_{\mathcal{T}}, L_{\alpha, \beta}\right)$}

In this section we will keep the set-up from last section so we can continue our investigation of the family of compact quantum metric spaces $\left(A_{\mathcal{T}}, L_{\alpha, \beta}\right)$ and study the limiting processes $\alpha=1, \beta \rightarrow 0$ and $\alpha \rightarrow 0, \beta=1$. There are limits in both cases, but they are of different nature. In the first case the expression $L_{1,0}$ has a an obvious meaning and it follows from (5) that this will be a seminorm on $A_{\mathcal{T}}$. This seminorm will be degenerate because its kernel will contain all of $C(P H)$, but on the other hand you can obtain the seminorm $L_{\mathcal{A}}$ directly from $L_{1,0}$, so we recover all the ingredients of the original spectral triple via this limit process. For the family $(\alpha, 1)$ with $\alpha$ decreasing from 1 to 0 
there is no sort of a limit on the level of seminorms, since $\alpha$ appears in the expression for $L_{\alpha, \beta}$ in the negative power $1 / \alpha$, but this does not affect the convergence of the corresponding compact quantum metric spaces since we prove that the spaces $\left(A_{\mathcal{T}}, L_{\alpha, 1}\right)$ converge to $\left(A_{\mathcal{C}}, L_{\mathcal{C}}\right)$ in the quantum Gromov-Hausdorff metric for $\alpha \rightarrow 0$. We have thought of possible interpretations of this result and do offer some remarks concerning the connection to physics in the text below, but we are not trained physicists, so we are reluctant to make too many comments in this direction.

The proofs of the results are based on some structural results on the dual space of a unital $\mathrm{C}^{*}$-algebra. Let the dual space of $\mathcal{T}$ be denoted $\mathcal{T}^{*}$, and we will then define two subspaces $\mathcal{N}$ and $\mathcal{S}$ of $\mathcal{T}^{*}$ by

$$
\begin{aligned}
\mathcal{N} & :=\left\{\phi \in \mathcal{T}^{*}|\|\phi \mid C(P H)\|=\|\phi\|\}\right. \\
\mathcal{S} & :=\left\{\phi \in \mathcal{T}^{*}|\|\phi \mid C(P H)\|=0\}\right.
\end{aligned}
$$

Here the letters $\mathcal{N}$ and $\mathcal{S}$ are chosen because they refer to the terms normal and singular functionals on $B(H)$. A priori it is not at all clear that $\mathcal{N}$ is a subspace, and we will not prove it here, but recall some results of Effros [14] which are presented just below. For details we refer to Dixmier's book [13] Proposition 2.11.7.

Proposition 4.1. With the notation described above, there exist positive contractive linear projection operators $N: \mathcal{T}^{*} \rightarrow \mathcal{N}$ and $S: \mathcal{T}^{*} \rightarrow$ $\mathcal{S}$ such that for any $\phi$ in $\mathcal{T}^{*}$

$$
\begin{gathered}
N(\phi)+S(\phi)=\phi \\
\|N(\phi)\|+\|S(\phi)\|=\|\phi\|
\end{gathered}
$$

It is easy to identify $\mathcal{N}$ with the dual space of $C(P H)$ simply by restricting a functional in $\mathcal{N}$ to $C(P H)$. The identification the other way goes via the fact that $B(P H)$ is the second dual of $C(P H)$, so the canonical embedding of $C(P H)^{*}$ into $C(P H)^{* * *}$ induces an embedding, say $\iota_{\mathcal{C}}$, of $C(P H)^{*}$ onto $\mathcal{N}$.

The space $\mathcal{S}$ may be identified with $\mathcal{A}^{*}$ in the following way. The identification is made via the homomorphism $\rho: \mathcal{T} \rightarrow \mathcal{A}$, which was defined in Definition 1.2. Any functional $\mu$ in $\mathcal{A}^{*}$ may be mapped into $\mathcal{S}$ by the composition $\mu \circ \rho$. Since the kernel of $\rho$ is $C(P H)$, it follows that this will be an isometric and order isomorphic mapping of $A^{*}$ onto $\mathcal{S}$, and we will denote this embedding $\iota_{\mathcal{A}}$.

As an immediate corollary of these identifications we get the following result. 
Corollary 4.2. For any state $\phi$ in $\mathcal{T}^{*}$ there exists a unique pair of states $f$ in $S(C(P H)$ ) and $\mu$ in $S(\mathcal{A})$ and a real $\alpha$ in $[0,1]$ such that $\phi=(1-\alpha) \iota_{C}(f)+\alpha \iota_{\mathcal{A}}(\mu)$.

These structures have been studied and generalized in [1], [2] and in the language of compact convex sets one would say that the two convex sets $\iota_{\mathcal{C}}(S(C(P H)))$ and $\iota_{\mathcal{A}}(S(\mathcal{A}))$ form a pair of split faces of $S(\mathcal{T})$.

The discussion on how the dual space of $C(P H)$ fits into the dual of $\mathcal{T}$ can be applied to the situation when $C(P H)$ is considered as a subalgebra of $\mathcal{C}=\widetilde{C(P H)}=C(P H)+\mathbb{C} I$ too. In this case we will fix a state $\sigma$ from the space $\mathcal{S}$ of singular functionals on $\mathcal{T}$ and use this state as a basis vector for the one-dimensional singular space associated to the decomposition of $\widetilde{C(P H)}{ }^{*}=\mathcal{N} \oplus \mathbb{C} \sigma$. In the general study of the variation of the metrics on $S(\mathcal{T})$ we will use $\sigma$ as a base point in the w*-compact space $S(\mathcal{T})$.

\section{The limit of $\left(A_{\mathcal{T}}, L_{1, \beta}\right)$ as $\beta \rightarrow 0$}

This limit is very easy to understand from the point of view of compact quantum metric spaces. It is simply an affine deformation at the level of seminorms as it can be seen immediately from the definitions 1.8 and 2.6. We will then extend that definition to cover the pair $(1,0)$ too, and let $L_{(1,0)}$ denote the corresponding seminorm. We can also still define the unit ball or Minkowski set $U_{(1,0)}$ for this seminorm by the definitions given at (18), and it follows that $A_{C} \cap C(P H)$ is contained in $U_{(1,0)}$. It is then easy to prove the following result.

Theorem 4.3. For any $a$ in $A_{\mathcal{A}}$ and $k$ in $A_{\mathcal{C}}$ :

$$
L_{1, \beta}(T(a)+k) \rightarrow L_{1,0}(T(a)+k)=L_{A}(a) \text { for } \beta \rightarrow 0
$$

For states $\phi, \psi$ on $\mathcal{T}$ with $\phi=\iota_{C}(f)+\iota_{A}(\mu), \psi=\iota_{C}(g)+\iota_{A}(\nu)$ the distance formula applied to the seminorm $L_{1,0}$ gives

$$
\operatorname{dist}_{1,0}(\phi, \psi)=\left\{\begin{array}{ll}
0 & \text { if } \phi=\psi \\
\infty & \text { if } f \neq g \\
\|\mu\| \operatorname{dist}_{\mathcal{A}}(\mu /\|\mu\|, \nu /\|\mu\|) & \text { if } f=g \text { and } \mu \neq \nu
\end{array} .\right.
$$

Proof. Since the kernel of $L_{(1,0)}$ contains all of $A_{C}$, it follows from the distance formula (8) that $\operatorname{dist}_{(1,0)}(\phi, \psi)=\infty$ if $f \neq g$. If $\phi \neq \psi$ and $f=g$ then $\|f\|=\|g\|<1$ so $\|\mu\|=\|\nu\|=1-\|f\| \neq 0$. Again the 
distance formula and (5) give right away that

$$
\begin{aligned}
\operatorname{dist}_{(1,0)}(\phi, \psi) & =\sup \left\{|(\phi-\psi)(t)| \mid L_{(1,0)}(t) \leq 1\right\} \\
& =\sup \left\{|(\mu-\nu)(a)| \mid L_{\mathcal{A}}(a) \leq 1\right\} \\
& =\|\mu\| \operatorname{dist}_{\mathcal{A}}(\mu /\|\mu\|, \nu /\|\mu\|) .
\end{aligned}
$$

We can not prove that the metric distances $\operatorname{dist}_{1, \beta}(\phi, \psi)$ converge to $\operatorname{dist}_{(1,0)}(\phi, \psi)$ for $\beta \rightarrow 0$, when the latter is finite, unless we have a trivial extension, but in the cases where the distance is infinite, $i$. e. when the normal parts, $f$ and $g$, of the states are different, we can always give an estimate of the speed of divergence.

Proposition 4.4. Let $0<\beta \leq 1$ be a real and $\phi, \psi$ states on $\mathcal{T}$ with decompositions $\phi=\iota_{C}(f)+\iota_{A}(\mu), \psi=\iota_{C}(g)+\iota_{A}(\nu)$. If $f \neq g$ then there exists a positive real $\gamma$ such that $\forall \beta \in(0,1]: \operatorname{dist}_{(1, \beta)}(\phi, \psi) \geq \gamma / \beta$.

Proof. We will establish a set theoretical inclusion from which the statement is easy to deduce.

$$
\frac{1}{\beta} U_{\mathcal{C}} \cap C(P H) \subseteq U_{1, \beta}
$$

This inclusion follows from the definitions presented in (16) and the computations which lead to (5). We can then see that the proposition follows when we define $\gamma$ by

$$
\gamma:=\sup \left\{|(f-g)(k)| \mid k \in U_{\mathcal{C}} \cap C(P H)\right\} .
$$

Suppose $\mathcal{A}$ is commutative and represents some classical system and $\mathcal{T}$ models a quantization of $\mathcal{A}$, then for a couple of states on $\mathcal{T}$, such as $\phi$ and $\psi$ we could look at $f, g$ as their quantum parts and $\mu, \nu$ as the classical parts. Then it appears that the limit for $\left.d_{(1, \beta}\right)(\phi, \psi)$ exists and gives the classical metric, scaled to the size of the classical parts if and only their quantum parts are identical. Another attempt to make an interpretation is that the inequality in the proposition above, implies that in a space where $\beta$ is small, the quantum parts are far apart; but we do not want to press this any further right now.

\section{The limit of $\left(A_{\mathcal{T}}, L_{\alpha, 1}\right)$ as $\alpha \rightarrow 0$}

We realized very early on that the family of compact quantum spaces $\left(A_{\mathcal{T}}, L_{\alpha, 1}\right)$ converges pointwise as concrete metric spaces towards 
$\left(\widetilde{C(P H)}, L_{\mathcal{C}}\right)$ when $\alpha$ decreases to 0 , but it took rather long to see that this convergence actually also works with respect to the quantum Gromov-Hausdorff metric. Before we prove this result we need a simple estimate.

Lemma 4.5. For any positive functional $f$ in the dual space $C(P H)^{*}$ :

$$
\sup \left\{|f(k)| \mid k \in U_{\mathcal{C}} \cap C(P H)\right\} \leq\|f\| \operatorname{diam}_{C} .
$$

Proof. Let $\varepsilon>0$ and choose $x$ in $U_{\mathcal{C}} \cap C(P H)$ such that $|f(x)| \geq$ $\sup \left\{|f(y)| \mid y \in U_{\mathcal{C}} \cap C(P H)\right\}-\varepsilon / 2$. Since $x$ is compact and $P H$ is of infinite dimension we can find a positive functional $g$ in $C(P H)^{*}$ such that $\|g\|=\|f\|$ and $|g(x)| \leq \varepsilon / 2$. Hence

$$
\|f\| \operatorname{diam}_{C} \geq|(f-g)(x)| \geq \sup \left\{|f(y)| \mid y \in U_{\mathcal{C}} \cap C(P H)\right\}-\varepsilon,
$$

Theorem 4.6. For $\alpha, \beta$ positive reals such that $\alpha \beta \leq 1$ :

$$
\operatorname{dist}_{q}\left(\left(A_{\mathcal{T}}, L_{\alpha, \beta}\right),\left(A_{\mathcal{C}}, \beta L_{\mathcal{C}}\right)\right) \leq \alpha\left(\operatorname{diam}_{\mathcal{A}}+\operatorname{diam}_{C}\right) .
$$

Proof. We will define a seminorm on $L$ on $A_{\mathcal{T}} \oplus A_{\mathcal{C}}$ which induces the given seminorms on each summand. Let $\sigma$ be a state on $\mathcal{T}$ which vanishes on $C(P H)$ and let $M$ be a big positive real number. We can then define the seminorm $L$.

$\forall a \in A_{\mathcal{A}} \forall k, h \in A_{\mathcal{C}} \cap C(P H) \forall s \in \mathbb{R}: L((T(a)+k, h+s I)):=$ $\max \left\{L_{(\alpha, \beta)}(T(a)+k), \beta L_{\mathcal{C}}(h), \frac{1}{\alpha} L_{\mathcal{A}}(a), \frac{1}{\alpha} L_{\mathcal{C}}(k-h), M|\sigma(T(a)-s I)|\right\}$

Let us show that the seminorm induced by $L$ on $A_{\mathcal{T}}$ is $L_{(\alpha, \beta)}$. By definition we always have $L((T(a)+k, h+s I)) \geq L_{(\alpha, \beta)}(T(a)+k)$ so it is enough to prove that for a given $t=T(a)+k$ with $a$ in $A_{\mathcal{A}}$ and $k$ in $A_{\mathcal{C}} \cap C(P H)$ we can find an $h$ in $A_{\mathcal{C}} \cap C(P H)$ and an $s$ in $\mathbb{R}$ such that $L((T(a)+k, h+s I))=L_{(\alpha, \beta)}(T(a)+k)$. We will prove that $h:=(1+\alpha \beta)^{-1} k$ and $s:=\sigma(T(a))$ will work. To this end we may without loss of generality assume that $L_{\alpha, \beta}(T(a)+k)=1$, and then by (22) it follows that $L_{\mathcal{A}}(a) \leq \alpha$, and by (23) we find that $L_{\mathcal{C}}(k) \leq$ $(1+\alpha \beta) / \beta$. From here it is easy to prove that $L(T(a)+k, h+s I)=1$. For the seminorm induced by $L$ on $A_{\mathcal{C}}$ we also get by definition that $L((T(a)+k, h+s I)) \geq \beta L_{C}(h)$. Let then an $h+s I$ be given in in $A_{\mathcal{C}}$ and define $a:=s I, k:=h$, then it is again a matter of computation to show that $L((T(a)+k, h+s I))=\beta L_{C}(h)$.

We will then show $\operatorname{dist}_{q}\left(\left(A_{\mathcal{T}}, L_{(\alpha, \beta)}\right),\left(A_{\mathcal{C}}, L_{\mathcal{C}}\right) \leq \alpha\left(\operatorname{diam}_{\mathcal{A}}+\operatorname{diam}_{C}\right)\right.$ by showing that for each positive $\varepsilon$ and any state $\phi$ on $\mathcal{T}$ there exists a state $\psi$ on $\widetilde{C(P H)}$ such that for the metric $\rho_{L}$ on the state space of 
$A_{\mathcal{T}} \oplus A_{\mathcal{C}}$ we have $\rho_{L}((\phi, 0),(0, \psi)) \leq \alpha\left(\operatorname{diam}_{\mathcal{A}}+\operatorname{diam}_{C}\right)+\varepsilon$, and vice versa.

For a state $\phi$ on $\mathcal{T}$ we can write $\phi=\iota_{\mathcal{C}}(f)+\iota_{\mathcal{A}}(\mu)$ for positive functionals $f$ on $C(P H)$ and $\mu$ on $\mathcal{A}$. Let $\hat{f}$ denote the extension - with the same norm - of $f$ to $\widehat{C(P H)}$, then the functional $\psi$ is defined as $\hat{f}+\|\mu\| \sigma$ on $\widetilde{C(P H)}$ and we get the following string of inequalities

$$
\begin{aligned}
& \rho_{L}((\phi, 0),(0, \psi)) \\
= & \sup \{|\phi(T(a)+k)-\psi(h+s I)| \mid L((T(a)+k, h+s I)) \leq 1\} \\
\leq & \sup \left\{|\phi(T(a))-\sigma(T(a))| \mid L_{\mathcal{A}}(a) \leq \alpha\right\} \\
+ & \sup \{|\sigma(T(a))-s||| \sigma(T(a))-s \mid \leq 1 / M\} \\
+ & \sup \left\{|f(k-h)| \mid L_{\mathcal{C}}(k-h) \leq \alpha\right\} \text { which by Lemma 4.5 } \\
\leq & \alpha\left(\operatorname{diam}_{\mathcal{A}}+\operatorname{diam}_{C}\right)+\frac{1}{M},
\end{aligned}
$$

Given a state $\psi$ on $\widetilde{C(P H)}$ we can write $\psi=\hat{f}+(1-\|f\|) \sigma$ for a positive functional $f$ on $C(P H)$ of norm at most 1 . Then the functional $\phi$ is defined as $\iota_{C}(f)+(1-\|f\|) \sigma$ on $\mathcal{T}$, and we get as above.

$$
\begin{aligned}
& \rho_{L}((\phi, 0),(0, \psi)) \\
= & \sup \{|\phi(T(a)+k)-\psi(h+s I)| \mid L((T(a)+k, h+s I)) \leq 1\} \\
\leq & \sup \left\{|\phi(T(a))-\sigma(T(a))| \mid L_{\mathcal{A}}(a) \leq \alpha\right\} \\
+ & \sup \{|\sigma(T(a))-s||| \sigma(T(a))-s \mid \leq 1 / M\} \\
+ & \sup \left\{|f(k-h)| \mid L_{\mathcal{C}}(k-h) \leq \alpha\right\} \\
\leq & \alpha\left(\operatorname{diam}_{\mathcal{A}}+\operatorname{diam}_{C}\right)+\frac{1}{M},
\end{aligned}
$$

and the theorem follows.

The inequalities just above show, that when $\alpha \rightarrow 0$ then the system seems to forget how it was created and only the very basic structure of the quantum infinitesimals modelled by $C(P H)$ are left visible.

\section{A QUANTUM METRIC ON THE SET OF PARAMETERS$$
\mathcal{P}:=\left\{(\alpha, \beta) \in \mathbb{R}^{2} \mid \alpha \geq 0, \beta>0, \alpha \beta \leq 1\right\} \cup\{(0, \infty)\}
$$

The quantum Gromov-Hausdorff metric on our two-parameter family of compact quantum Hausdorff spaces naturally define a metric on the parameter space, say $\mathcal{P}^{\circ}:=\left\{(\alpha, \beta) \in \mathbb{R}_{+}^{2} \mid \alpha \beta \leq 1\right\}$, and we want to get an impression on the sort of metric space we can obtain this way. We have not made a very detailed study of this but we show that 
some balls in this metric are unbounded with respect to the Euclidian distance in $\mathbb{R}^{2}$. We also show, the other way around, that some sets which are bounded with respect the Euclidian metric are unbounded with respect to the quantum-metric. Based on the results in Theorem 4.6 we realized that it is reasonable to extend the parameter space to the space $\mathcal{P}$, defined below.

$$
\begin{aligned}
\mathcal{P} & :=\{(\alpha, \beta) \mid \alpha \geq 0, \beta>0, \alpha \beta \leq 1\} \cup\{(0, \infty)\} \\
\text { for } 0<\beta<\infty & :\left(A_{0, \beta}, L_{0, \beta}\right):=\left(A_{\mathcal{C}}, \beta L_{\mathcal{C}}\right) \\
\left(A_{0, \infty}, L_{0, \infty}\right) & :=(\mathbb{R}, 0) .
\end{aligned}
$$

The points we have added are also compact quantum metric spaces, and it turns out that they fit in very well with respect to the quantum Gromov-Hausdorff metric.

Proposition 5.1. Let $\beta_{0}>0$ then the subset $\mathcal{P}_{\beta_{0}}:=\{(\alpha, \beta) \in \mathcal{P} \mid \beta \geq$ $\left.\beta_{0}\right\}$ is compact with respect to the metric inherited from the quantum Gromov-Hausdorff distance.

Proof. Fix a positive $\varepsilon$ and define $\beta_{1}:=\max \left\{\beta_{0}, 2\left(\operatorname{diam}_{A}+\operatorname{diam}_{C}\right) / \varepsilon\right\}$. For any pair $(\alpha, \beta)$ in $\mathcal{P}$ with $\beta \geq \beta_{1}$ we get $\alpha \leq \beta_{1}^{-1}$ and by Theorem 4.6

$$
\operatorname{dist}_{q}((\alpha, \beta),(0, \beta)) \leq \alpha\left(\operatorname{diam}_{A}+\operatorname{diam}_{C}\right) \leq\left(\operatorname{diam}_{A}+\operatorname{diam}_{C}\right) / \beta_{1} .
$$

By Proposition 3.8

$$
\operatorname{dist}_{q}((0, \beta),(0, \infty)) \leq \operatorname{diam}_{C} / \beta \leq \operatorname{diam}_{C} / \beta_{1} .
$$

hence it follows that for $(\alpha, \beta)$ in $\mathcal{P}$ with $\beta \geq \beta_{1}$ this point is in the ball of radius $\varepsilon$ with centre in $(0, \infty)$. We are then left with the set $\left\{(\alpha, \beta) \in \mathcal{P}_{\beta_{0}} \mid \beta_{0} \leq \beta \leq \beta_{1}\right\}$ and we will divide this set into two sets dependent on a positive real $\delta$ which we define by

$$
\delta:=\min \left\{\frac{\varepsilon}{3\left(1+\operatorname{diam}_{\mathcal{A}}+\operatorname{diam}_{C}\right)}, \frac{1}{\beta_{1}}\right\}
$$

and the sets become

$$
\begin{aligned}
& \mathcal{X}:=\left\{(\alpha, \beta) \mid 0 \leq \alpha \leq \delta \text { and } \beta_{0} \leq \beta \leq \beta_{1}\right\} \\
& \mathcal{Y}:=\left\{(\alpha, \beta) \mid \delta \leq \alpha \text { and } \beta_{0} \leq \beta \leq \beta_{1} \text { and } \alpha \beta \leq 1\right\}
\end{aligned}
$$

The results from Theorem 3.9 show that the usual Euclidian metric and the metric dist $_{q}$ generate the same topology on the subset $\mathcal{Y}$, so this set is compact. For the set $\mathcal{X}$ we can look at the subset $\mathcal{Z}$ which we define by

$$
\mathcal{Z}:=\left\{(\delta, \beta) \mid \beta_{0} \leq \beta \leq \beta_{1}\right\}
$$


Since $\mathcal{Z}$ is also a subset of $\mathcal{Y}$, it is compact for the quantum metric dist $_{q}$, by the result above, and we can find a finite number of points $\left\{\left(\delta, \beta_{i}\right) \mid i \in J\right\}$ in $\mathcal{Z}$ such that any point in $\mathcal{Z}$ is within distance $\delta$ from a point of the form $\left(\delta, \beta_{i}\right)$. For any point $(\alpha, \beta)$ in $\mathcal{X}$, we get from Theorem 4.6 that $\operatorname{dist}_{q}((\alpha, \beta),(0, \beta)) \leq \varepsilon / 3$ and for suitable $\beta_{i}$ we get

$$
\begin{aligned}
\operatorname{dist}_{q}\left((\alpha, \beta),\left(\delta, \beta_{i}\right)\right) & \leq \operatorname{dist}_{q}((\alpha, \beta),(0, \beta)) \\
& +\operatorname{dist}_{q}((0, \beta),(\delta, \beta))+\operatorname{dist}_{q}\left((\delta, \beta),\left(\delta, \beta_{i}\right)\right) \leq \varepsilon,
\end{aligned}
$$

and the proposition follows.

We will then look at the subsets of $\mathcal{P}$ such that $\alpha \geq \alpha_{0}$. Here the situation is quite the opposite since these sets will be unbounded with respect to the quantum metric on $\mathcal{P}$. To see this we fix a positive $\gamma \leq 1$ and we will study behavior of the metric along the hyperbola $\mathcal{H}_{\gamma}:=$ $\left\{(\alpha, \beta) \in \mathbb{R}_{+}^{2} \mid \alpha \beta=\gamma\right\}$. We see that the seminorms corresponding to the points on $\mathcal{H}_{\gamma}$ are all proportional and for any positive real $s$ we see from the Definition 2.6 $L_{(\gamma / s), s}=s L_{\gamma, 1}$, so the space is well understood along each of these curves. In particular, for the diameters we have $\operatorname{diam}_{(\gamma / s), s}=\left(\operatorname{diam}_{\gamma, 1}\right) / s$, so for $s \leq 1$ and $s$ decreasing to 0 , we get immediately the following estimate.

Proposition 5.2. For positive reals $\gamma, s$ such that $0<\gamma \leq 1$ and $0<s \leq 1 / \gamma$ :

$$
\operatorname{dist}_{q}\left(\left(A_{\mathcal{T}}, L_{\gamma, 1}\right),\left(A_{\mathcal{T}}, L_{(\gamma / s), s}\right)\right) \geq(1 / 2)\left(s^{-1}-1\right) \operatorname{diam}_{\gamma, 1}
$$

Proof. Let $\varepsilon>0$ and let $\delta:=\operatorname{dist}_{q}\left(\left(A_{\mathcal{T}}, L_{\gamma, 1}\right),\left(A_{\mathcal{T}}, L_{(\gamma / s), s}\right)\right)$. For a pair of states, say $\phi, \psi$ on $\mathcal{A}_{\mathcal{T}}$ such that $d_{(\gamma / s), s}(\phi, \psi) \geq\left(\operatorname{diam}_{\gamma, 1}\right) / s-\varepsilon / 3$ we can find approximating states - with respect to $\left(A_{\mathcal{T}}, L_{\gamma, 1}\right)$, - say $\mu$ and $\nu$ on $\mathcal{A}_{\mathcal{T}}$ such that

$$
\begin{aligned}
\left(\operatorname{diam}_{\gamma, 1}\right) / s-\varepsilon / 3 & \leq \operatorname{dist}_{(\gamma / s), s}(\phi, \psi) \\
& \leq 2 \delta+2 \varepsilon / 3+\operatorname{dist}_{\gamma, 1}(\mu, \nu) \\
& \leq 2 \delta+2 \varepsilon / 3+\operatorname{diam}_{\gamma, 1},
\end{aligned}
$$

and the proposition follows.

For the vertical intervals $\{(\alpha, \beta) \mid 0<\beta \leq \alpha\}$ we get that they are all unbounded with respect to this new metric. This follows easily from Proposition 4.4, and we will state it formally in the following proposition. 
Proposition 5.3. For a fixed $\alpha_{0}>0$ there exists a positive $\gamma$ such that

$$
\left.\forall \beta \in] 0,1 / \alpha_{0}\right]: \operatorname{diam}_{\alpha_{0}, \beta} \geq \gamma / \beta \text {. }
$$

\section{Applications to the Compacts and an investigation of CONNES' 7 AXIOMS FOR THIS SPECTRAL TRIPLE.}

Right after the Definition 1.1 of the Toeplitz extension of a $\mathrm{C}^{*}$ algebra $\mathcal{A}$, we remarked that it is debatable if the generalized Toeplitz algebra should be defined as the $\mathrm{C}^{*}$-algebra generated by $P \mathcal{A} \mid P H$ alone or - as we have chosen - the one generated by this set plus the compacts. The difference is a trivial extension, but for the algebra $\widehat{C(H)}$ it is a rather crucial difference, when this algebra is considered to be a trivial extension of the one dimensional $\mathrm{C}^{*}$-algebra $\mathbb{C} I$. Our first example here shows that our construction offers a variety of spectral triples for the unitarized compacts. On the other hand, for the Podles̀ sphere our construction gives an algebra which has more compacts than the universal $\mathrm{C}^{*}$-algebra for the Podles̀ sphere has. If we just had used the $\mathrm{C}^{*}$-algebra generated by $P \mathcal{A} \mid P H$, we would have obtained the right algebra here.

Example 6.1. Let $H$ be a separable infinite dimensional Hilbert space and let $\mathcal{A}:=\mathbb{C} I$ be the unital $C^{*}$-algebra generated the unit $I$ on $H$. Let $D$ be an unbounded self-adjoint invertible operator on $H$ with compact inverse and let the projection $P:=I$. We now have a spectral triple $(\mathcal{A}, H, D)$ and a quadruple $((\mathcal{A}, H, D), P)$ of Toeplitz type. Our construction will then give a $C^{*}$-algebra $\mathcal{T}:=\widehat{C(H)}$, a Hilbert space $K:=H \oplus H$ and a representation $\pi$ of $\mathcal{T}$ on $K$ by

$$
\forall k+\gamma I \in C(H)+\mathbb{C} I \quad \pi(k+\gamma I):=\left(\begin{array}{cc}
k+\gamma I & 0 \\
0 & \gamma I
\end{array}\right) .
$$

The Dirac operator then becomes

$$
D_{\alpha, \beta}:=\left(\begin{array}{cc}
0 & \beta D \\
\beta D & \frac{1}{\alpha} D
\end{array}\right)
$$

You may notice that the part $1 / \alpha D$ has no effect, for this spectral triple and this leads to the following proposition which will yield many more spectral triples associated to $\widehat{C(H)}$.

Proposition 6.2. Based on the notation in the example above let $T$ be an unbounded densely defined and closed operator on $H$. If $|T|$ is 
invertible with compact resolvent then for

$$
D:=\left(\begin{array}{cc}
0 & T^{*} \\
T & 0
\end{array}\right)
$$

the set $\left(|T|^{-1} C(H)|T|^{-1}+\mathbb{C} I, K, D\right)$ is a spectral triple associated to $\widetilde{C(H)}$.

In the article [8] Connes lists 7 axioms for Non Commutative Geometry. In the present case of a spectral triple associated to the unitarized compacts $\widehat{C(H)}$ the dimension must be 0 so the dimension is even and for the Dirac operator $D$ the growth of the eigenvalues must be such that for any positive real $s$ the operator $\left(I+D^{2}\right)^{-s / 2}$ is of trace class. There should also be a grading $\gamma$ and a conjugate linear operator $J$ which relate in certain ways. We can provide candidates for these ingredients, which seem natural to us, but they will not fulfill all of Connes' axioms. We will therefore present the candidates for $D, \gamma, J$, check each of the axioms and show what sort of problems we are facing. We keep the notation from above in this section and define

\section{Definition 6.3.}

(D) Let $T$ be a self-adjoint unbounded operator with trivial kernel, such that for any positive real $s$ the operator $|T|^{-s}$ is of trace class, then the Dirac operator $D$ is defined on $H \oplus H$ by

$$
D:=\left(\begin{array}{cc}
0 & T \\
T & 0
\end{array}\right)
$$

$(\gamma)$ The obvious choice for this unitary seems to be the unitary on $H \oplus H$, given by

$$
\gamma:=\left(\begin{array}{cc}
I & 0 \\
0 & -I
\end{array}\right) .
$$

(J) It is not so obvious what to choose here, since our setup is not the same as the one Connes clearly has in mind. In [8] Connes obtains the $J$ operation from a standard representation of a selfadjoint algebra of bounded operators. This is not what we have here for $C(H)$, but we have anyway a candidate for $J$ which seems reasonable. First we define $j: H \rightarrow H$ by chosing an orthonormal basis $\left(\xi_{n}\right)$ for $H$ consisting of eigenvectors for $T$. Then we define $j$ on $\lambda \xi_{n}$ as $\bar{\lambda} \xi_{n}$ and extend this to a conjugate linear isometry of $H$ onto $H$. The choice for $J$ is then given by

$$
J:=\left(\begin{array}{ll}
0 & j \\
j & 0
\end{array}\right) .
$$


Remark that

$$
J \pi\left(a^{*}+\bar{\lambda} I\right) J=\left(\begin{array}{cc}
j a^{*} j+\lambda I & 0 \\
0 & \lambda I
\end{array}\right)
$$

so for any operators $a+\lambda I$ and $b+\mu I$ the operators $\pi(a+\lambda I)$ and $J \pi\left(b^{*}+\bar{\mu} I\right) J$ do commute.

We will then look at the 7 axioms taken from [8] one by one, but first we will define the algebra $\mathcal{A}$ of smooth elements as the operators $a \in \widetilde{C(H)}$ such that for $\delta(x):=[|D|, x]$ we have for any $a \in \mathcal{A}$ and any natural number $m$ both $\pi(a)$ and $[D, \pi(a)]$ are in the domain of $\delta^{m}$. Inside this algebra $\mathcal{A}$ we have a norm dense subalgebra of operators of finite rank which we denote $\mathcal{A}_{0}$. This algebra is defined via the orthonormal basis $\left(\xi_{n}\right)$, from above, consisting of eigenvectors for $T$. The algebra $\mathcal{A}_{0}$ is then the linear span of the matrix units $a_{i j}:=\left\langle., \xi_{j}\right\rangle \xi_{i}$.

(1) The operator $D^{-1}$ is an infinitesimal of infinite order. This is fulfilled by the demand that $|T|^{-s}$ is of trace class for any psoitive real $s$.

(2) For any pair of elements $a, b \in \mathcal{A}:\left[[D, \pi(a)], J \pi\left(b^{*}\right) J\right]=0$. This demand can not be met, but we have - as in Dabrowski's paper [12] - $\forall a, b \in \mathcal{A}:\left[[D, \pi(a)], J \pi\left(b^{*}\right) J\right] \in C(H \oplus H)$.

(3) Smoothness The smoothness axiom is fulfilled by the definition of the algebra $\mathcal{A}$.

$(4,5,6)$ We can not show that the spectral triple we investigate fulfills any of these 3 axioms. We only have the grading $\gamma$ and it seems to be uniquely determined by its basic properties.

(7) We have an operator $J$ such that $\left[\pi(a), J \pi\left(b^{*}\right) J\right]=0$, but $\gamma$ and $J$ do not fit with the reality properties of the table in [8] p. 162. We get $J^{2}=I, J D=D J, J \gamma=-\gamma J$. For $n=0$ the first two identities are as in the table, but the last one should have been $J \gamma=\gamma J$.

We will now turn to the Podles̀ sphere, $C\left(S_{q c}^{2}\right)$, [25] and base the presentation here on Section 4 of that paper and on Chakraborty's description, [6], of a concrete faithful representation for this algebra. Chakraborty's purpose was to some extent the same as ours since he wanted to create a Lip-norm for the Podles̀ sphere, based on the fact that this $\mathrm{C}^{*}$-algebra is an extension of the classical Toeplitz algebra by the compacts. In the latter presentation the parameter $\mu$ from Podles̀ paper is replaced by the letter $q$, which now seems to be standard, so we will use this notation. 
For $c, q$ reals such that $c>0$ and $0<|q|<1$ the Podles̀ sphere, $C\left(S_{q c}^{2}\right)$ is the universal $C^{*}$-algbera generated by 2 operators $A$ and $B$ which satisfy the following relations:

$$
A=A^{*}, B A=q^{2} A B, B^{*} B=A-A^{2}+c I, B B^{*}=q^{2} A-q^{4} I+c I .
$$

Let $\mathcal{T}(\mathbb{T})$ denote the classical Toeplitz algebra for the unit circle and let $\rho: \mathcal{T}(\mathbb{T}) \rightarrow C(\mathbb{T})$ denote the canonical surjective homomorphism. All the algebras $C\left(S_{q c}^{2}\right)$ turns out to be isomorphic [29] and can be described by

$$
C\left(S_{q c}^{2}\right)=\{(x, y) \in \mathcal{T}(\mathbb{T}) \oplus \mathcal{T}(\mathbb{T}) \mid \rho(x)=\rho(y)\} .
$$

By So we can see that $C\left(S_{q c}^{2}\right)$ is an extension of the Toeplitz algebra by the compacts.

Let us consider the standard spectral triple associated to the $\mathrm{C}^{*}$ algebra $\mathcal{A}$ of continuous functions on the unit circle. For the algebra $A$ we can take the functions whose Fourier coefficients form rapidly decreasing sequences and the Dirac operator is $\frac{1}{i} \frac{d}{d \theta}$. As the Hilbert space $H$ we take $L^{2}(\mathbb{T})$ and the projection $P$ is the projection onto $H_{+}$, the closed linear span of the eigen functions $e^{i n \theta}, n>0$, corresponding to the positive eigen values. This is not the usual definition, where the constant function $I$ usually is assumed to be in $H_{+}$. This will not change the construction qualitatively but it will have the nice consequence, that the restriction of $D$ to $H_{+}$is invertible with a compact inverse. Finally we let $H_{-}$denote the orthogonal complement of $H_{+}$in $H$. The quadruple $((\mathcal{A}, H, D), P)$ is then of Toeplitz type and the Definition 1.8 gives a spectral triple $\left(A_{t}, K, D_{\alpha, \beta}\right)$ for the ordinary Toeplitz algebra. Recall that the Hilbert space $K$ is given as $H_{+} \oplus H_{+} \oplus H_{-}$, so we can define a projection $Q$ of $K$ onto the first two summands and it follows from Definition 1.8,(v), that $D_{\alpha, \beta}$ commutes with $Q$. By checking the same definition's point (iv) it can be seen that for any $t$ in $\mathcal{T}(\mathbb{T})$ the commutator $[\pi(t), Q]$ is compact since it is nothing but the embedding of the operator $[\rho(t), P]$ into $B(K)$. In order to show, that we now have a quadruple of Toeplitz type in the set $\left(\left(A_{t}, K, D_{\alpha, \beta}\right), Q\right)$, we then, according to Definition 1.2 only have to prove that $D_{(\alpha, \beta) Q}:=D_{\alpha, \beta} \mid Q K$ has trivial kernel, but this follows easily from the description of $D_{\alpha, \beta}$ given in Definition 1.8 point $(\mathrm{v})$ and the fact that $D_{P}$ is assumed to be injective. As mentioned above we will not consider the extended algebra as defined in Definition 1.1. Instead we will define $\mathcal{T}_{\mathcal{T}}$ as the $\mathrm{C}^{*}$-algebra on $H_{+} \oplus H_{+}$generated by $Q \pi(t) \mid Q K$. It is not difficult to see that this $\mathrm{C}^{*}$-algebra is exactly the one which above is described as the algebra $C\left(S_{q c}^{2}\right)$. Our construction can give a family of spectral 
triples associated to $C\left(S_{q c}^{2}\right)+C\left(H_{+} \oplus H_{+}\right)$, and let $\left(A_{t t}, K_{t t}, D_{t t}\right)$ denote such a set. Then we are left with the problem to realize how the algebra $A_{t t}$ relates to the sum $C\left(S_{q c}^{2}\right)+C\left(H_{+} \oplus H_{+}\right)$. To deal with this question we first remark that by Definition 1.8 point (ii) any element in $A_{t t}$ is a sum of an element related to a differentiable symbol and a differentiable compact. Consequently we only have to see how a differentiable compact, say $C$ in $C\left(H_{+} \oplus H_{+}\right)$) behaves with respect to the splitting as a sum of a diagonal operator and an off diagonal operator,

$$
C=\left(\begin{array}{ll}
u & v \\
x & y
\end{array}\right)=\left(\begin{array}{ll}
u & 0 \\
0 & y
\end{array}\right)+\left(\begin{array}{ll}
0 & v \\
x & 0
\end{array}\right) .
$$

By Definition 1.8 point (i) the matrix above is a differentiable compact if and only if both of the products $D_{(\alpha, \beta) Q} C$ and $C D_{(\alpha, \beta) Q}$ are bounded and densely defined. In matrix forms these products are as seen below

$$
\begin{aligned}
D_{(\alpha, \beta) Q} C & =\left(\begin{array}{cc}
\beta D_{P} x & \beta D_{P} y \\
\beta D_{P} u+\frac{1}{\alpha} D_{P} x & \beta D_{P} v+\frac{1}{\alpha} D_{P} y
\end{array}\right) \\
C D_{(\alpha, \beta) Q} & =\left(\begin{array}{ll}
\beta v D_{P} & \beta u D_{P}+\frac{1}{\alpha} v D_{P} \\
\beta y D_{P} & \beta x D_{P}+\frac{1}{\alpha} y D_{P}
\end{array}\right) .
\end{aligned}
$$

From here it follows that the products $D_{(\alpha, \beta) Q} C$ and $C D_{(\alpha, \beta) Q}$ are bounded and densely defined if and only if all of the operators $u, v, x, y$ belong to the algebra $A_{c}$, as defined in Definition 1.8 point (i). This has the immediate consequence that if we replace $A_{t t}$ by $A_{t t d}$ which we define by

$$
A_{t t d}:=\left\{\left(\begin{array}{ll}
a & 0 \\
0 & d
\end{array}\right) \mid\left(\begin{array}{ll}
a & 0 \\
0 & d
\end{array}\right) \in A_{t t}\right\},
$$

then $\left(A_{t t d}, K_{t t}, D_{t t}\right)$ is a spectral triple associated to the universal C*algebra for the Podles̀ sphere.

Remark 6.4. We have been asked by the referee, if there are some connections between our example of a spectral triple for the Podlès sphere and the ones obtained in [10] and [11]. We have, but in vain, tried to answer this question, and it is our impression that there is no simple connection.

\section{Odd And EVEn EXTEnsions an AnAlytic K-Homology}

Our constructions in this paper produce odd spectral triples and this seems not to be the right setup for algebras containing the compact operators. It is quite easy to produce an even spectral triple from 
an odd one by doubling the representation and introduce the Dirac operator $\hat{D}$ on the Hilbert space $K \oplus K$ which is given by

$$
\hat{D}=\left(\begin{array}{cc}
0 & D \\
D & 0
\end{array}\right)
$$

The grading $\gamma$ is then given on $K \oplus K$ by $\gamma(\xi, \eta):=(\xi,-\eta)$. We could have performed all our computations in this setting, but it would not give any new insights with respect to the metric properties we have been investigating in this article, so we have not pursued a presentation this way.

Extensions of unital $\mathrm{C}^{*}$-algebras by the compacts as we do it here is described in Higson and Roe's book [16] Chapter 5. So according to that description an extension of the sort we are looking at corresponds to an element in the reduced analytic K-homology of the unital $\mathrm{C}^{*}$ algebra $\mathcal{A}$. But our construction is only designed for projections $P$ in the commutant of $D$.

\section{REFERENCES}

[1] E. M. Alfsen. Compact convex sets and boundary integrals. Springer-Verlag, 1971.

[2] E. M. Alfsen, F. W. Shultz. State spaces of operator algebras. Basic theory, orientations and $C^{*}$-products, Birkhuser, 2001.

[3] S. Baaj, P. Julg, Théorie bivariante de Kasparov et opérateurs non bornés dans les $C^{*}$-modules hilbertiens, C.R. Acad. Sci. Paris, Serie I, 296 (1983), 875-878.

[4] I. Belgradek, Degenerations of Riemannian manifolds, arXiv:mathDG /0701723, 2007.

[5] D. Burago, Y. Burago, S. Ivanov, A course in metric geometry, Graduate Studies in Mathematics, 33. American Mathematical Society, Providence, RI, 2001.

[6] P. S. Chakraborty. From $C^{*}$-algebra extensions to $C Q M S, S U_{q}(2)$, Podlè̀ sphere and other examples. arXiv:math/02100155v1.

[7] A. Connes. Non Commutative Geometry. Academic Press, San Diego, 1994.

[8] A. Connes, Gravity coupled with matter and the foundation of non commutative geometry, Comm. Math. Phys. 182 (1996), 155-176.

[9] A. Connes, H. Moscovici, Transgression and the Chern character of finitedimensional K-cycles, Comm. Math. Phys. 155 (1993), 103-122.

[10] L. Dąbrowski, F. D'Andrea, G. Landi, E. Wagner, Dirac operators on all Podleś quantum spheres, J. Noncommut. Geom., 1 (2007), 213-239.

[11] L. Dąbrowski, A. Sitarz, Dirac operator on the standard Podleś quantum sphere, Noncommutative geometry and quantum groups (Warsaw, 2001), 49-58, Banach Center Publ., 61, Polish Acad. Sci., Warsaw, 2003.

[12] K. R. Davidson, $C^{*}$-algebras by example, Fields Institute Monographs, 6, American Mathematical Society, Providence, RI, 1996.

[13] J. Dixmier. Les $C^{*}$-algèbres et leurs représentations, Gauthier-Villars, Paris, 1964. 
[14] E. G. Effros, Order ideals in a $C^{*}$-algebra and its dual, Duke Math. J., 30 (1963), 391-412.

[15] K. Fukaya, Metric Riemannian Geometry, Handbook of differential geometry, Vol. II, Elsevier/North-Holland, Amsterdam, 2006, 189-313.

[16] N.Higson, J. Roe, Analytic K-Homology, Oxford University Press, Oxford, 2000.

[17] D. Kerr, Matricial quantum Gromov-Hausdorff distance J. Funct. Anal., 205 (2003), 132-167.

[18] D. Kerr, H. Li On Gromov-Hausdorff convergence for operator metric spaces, arXiv: mathOA/0411157 v4, 2007.

[19] S. Klimek, A. Lesniewski, A two-parameter quantum deformation of the unit disc, J. Funct. Anal., 115 (1993), 1-23.

[20] S. Klimek, A. Lesniewski, Quantum Riemann surfaces. I. The unit disc, Comm. Math. Phys., 146 (1992), 103-122.

[21] F. Latremoliere, Approximation of quantum tori by finite quantum tori for the quantum Gromov-Hausdorff distance, J. Funct. Anal. 223 (2005), 336395.

[22] H. Li, Order-unit quantum Gromov-Hausdorff distance, J. Funct. Anal. 231 (2006), 312-360.

[23] G. Nagy, On the Haar measure of the quantum $\mathrm{SU}(N)$ group, Comm. Math. Phys., 153 (1993), 217-228.

[24] G.K. Pedersen, Analysis now, Graduate Texts in Mathematics, 118, Springer-Verlag, New York, 1989.

[25] P. Podles̀, Quantum spheres, Lett. Math. Phys. 14 1987, 193-202.

[26] M. A. Rieffel, Leibniz seminorms for "matrix algebras converge to the sphere", arXiv:mathOA/0707.3229, 2007.

[27] M. A. Rieffel, Gromov-Hausdorff distance for quantum metric spaces, Mem. Amer. Math. Soc., 168 (2004), no. 796, 1-65.

[28] M. A. Rieffel Metrics on state spaces, Doc. Math. 4 (1999), 559-600.

[29] A. J. L. Sheu, Quantization of the Poisson SU(2) and its Poisson homogeneous space-the 2-sphere. With an appendix by Jiang-Hua Lu and Alan Weinstein, Comm. Math. Phys., 135 (1991), 217-232.

[30] Wu. W, Quantized Gromov-Hausdorff distance, J. Funct. Anal. 238 (2006), $58-98$.

Department of Mathematics, University of Copenhagen, DK-2100

Copenhagen, Denmark

E-mail address: echris@math.ku.dk

Department of Mathematics, Leibniz University of Hannover, 30167

Hannover, Germany

E-mail address: ivan@math.uni-hannover.de 